\title{
Unraveling the polygenic architecture of complex traits using blood eQTL meta- analysis
}

Urmo Võsa*\#1,2, Annique Claringbould ${ }^{* \# 1}$, Harm-Jan Westra**1, Marc Jan Bonder**1, Patrick Deelen $^{* * 1,3}$, Biao Zeng ${ }^{4}$, Holger Kirsten ${ }^{5}$, Ashis Saha ${ }^{6}$, Roman Kreuzhuber ${ }^{7,8}$, Silva Kasela ${ }^{2}$, Natalia Pervjakova $^{2}$, Isabel Alvaes ${ }^{9}$, Marie-Julie Fave ${ }^{9}$, Mawusse Agbessi ${ }^{9}$, Mark Christiansen ${ }^{10}$, Rick Jansen ${ }^{11}$, Ilkka Seppälä ${ }^{12}$, Lin Tong ${ }^{13}$, Alexander Teumer ${ }^{14}$, Katharina Schramm ${ }^{15,16}$, Gibran Hemani ${ }^{17}$, Joost Verlouw ${ }^{18}$, Hanieh Yaghootkar ${ }^{19}$, Reyhan Sönmez ${ }^{20,21}$, Andrew Brown ${ }^{22,23,21}$, Viktorija Kukushkina ${ }^{2}$, Anette Kalnapenkis ${ }^{2}$, Sina Rüeger ${ }^{24}$, Eleonora Porcu ${ }^{24}$, Jaanika KronbergGuzman², Johannes Kettunen ${ }^{25}$, Joseph Powell ${ }^{26}$, Bernett Lee ${ }^{27}$, Futao Zhang ${ }^{28}$, Wibowo Arindrarto $^{29}$, Frank Beutner ${ }^{30}$, BIOS Consortium, Harm Brugge ${ }^{1}$, i2QTL Consortium, Julia Dmitreva $^{31}$, Mahmoud Elansary ${ }^{31}$, Benjamin P. Fairfax ${ }^{32}$, Michel Georges ${ }^{31}$, Bastiaan T. Heijmans $^{29}$, Mika Kähönen ${ }^{33}$, Yungil Kim ${ }^{34,35}$, Julian C. Knight ${ }^{32}$, Peter Kovacs ${ }^{36}$, Knut Krohn ${ }^{37}$, Shuang Li ${ }^{1}$, Markus Loeffler ${ }^{5}$, Urko M. Marigorta ${ }^{4}$, Hailang Mei ${ }^{38}$, Yukihide Momozawa ${ }^{31,39}$, Martina Müller-Nurasyid ${ }^{15,16,40}$, Matthias Nauck ${ }^{41}$, Michel Nivard ${ }^{42}$, Brenda Penninx ${ }^{11}$, Jonathan Pritchard ${ }^{43}$, Olli Raitakari ${ }^{44}$, Olaf Rotzchke ${ }^{27}$, Eline P. Slagboom ${ }^{29}$, Coen D.A. Stehouwer ${ }^{45}$, Michael Stumvoll ${ }^{46}$, Patrick Sullivan ${ }^{47}$, Peter A.C. 't Hoen ${ }^{48}$, Joachim Thiery ${ }^{49}$, Anke Tönjes ${ }^{46}$, Jenny van Dongen ${ }^{11}$, Maarten van Iterson ${ }^{29}$, Jan Veldink ${ }^{50}$, Uwe Völker ${ }^{51}$, Cisca Wijmenga ${ }^{1}$, Morris Swertz ${ }^{3}$, Anand Andiappan ${ }^{27}$, Grant W. Montgomery ${ }^{52}$, Samuli Ripatti ${ }^{53}$, Markus Perola ${ }^{54}$, Zoltan Kutalik ${ }^{24}$, Emmanouil Dermitzakis ${ }^{22,23,21}$, Sven Bergmann ${ }^{20,21}$, Timothy Frayling ${ }^{19}$, Joyce van Meurs ${ }^{18}$, Holger Prokisch $^{55,56}$, Habibul Ahsan ${ }^{13}$, Brandon Pierce ${ }^{13}$, Terho Lehtimäki ${ }^{12}$, Dorret Boomsma ${ }^{11}$, Bruce M. Psaty ${ }^{10,57}$, Sina A. Gharib ${ }^{58,10}$, Philip Awadalla ${ }^{9}$, Lili Milani ${ }^{2}$, Willem Ouwehand ${ }^{7,59}$, Kate Downes ${ }^{7}$, Oliver Stegle $8,60,61$, Alexis Battle ${ }^{62}$, Jian Yang ${ }^{28,63}$, Peter M. Visscher ${ }^{28}$, Markus Scholz ${ }^{5}$, Gregory Gibson ${ }^{4}$, Tõnu Esko², Lude Franke\#1

Department of Computer Science, Johns Hopkins University, Baltimore, United States of America

7. Department of Haematology, University of Cambridge and NHS Blood and Transplant Cambridge Biomedical Campus, Cambridge, United Kingdom

8. European Molecular Biology Laboratory, European Bioinformatics Institute, Wellcome Genome Campus, Hinxton, Cambridge CB10 1SD, United Kingdom

9. Computational Biology, Ontario Institute for Cancer Research, Toronto, Canada

10. Cardiovascular Health Research Unit, University of Washington, Seattle, United States of America

11. Vrije Universiteit Amsterdam, Amsterdam, The Netherlands

12. Department of Clinical Chemistry, Fimlab Laboratories and Faculty of Medicine and Life Sciences, University of Tampere, Tampere, Finland

13. Department of Public Health Sciences, University of Chicago, Chicago, United States of America

14. Institute for Community Medicine, University Medicine Greifswald, Greifswald, Germany

15. Institute of Genetic Epidemiology, Helmholtz Zentrum München - German Research Center for Environmental Health, Neuherberg, Germany

16. Department of Medicine I, University Hospital Munich, Ludwig Maximilian's University, München, Germany 
bioRxiv preprint doi: https://doi.org/10.1101/447367; this version posted October 19,2018 . The copyright holder for this preprint (which was not certified by peer review) is the author/funder. All rights reserved. No reuse allowed without permission.

17. MRC Integrative Epidemiology Unit, University of Bristol, Bristol, United Kingdom

18. Department of Internal Medicine, Erasmus Medical Centre, Rotterdam, The Netherlands

19. Exeter Medical School, University of Exeter, Exeter, United Kingdom

20. Department of Computational Biology, University of Lausanne, 1015 Lausanne, Switzerland

21. Swiss Institute of Bioinformatics, 1015 Lausanne, Switzerland

22. Department of Genetic Medicine and Development, University of Geneva Medical School, Geneva, Switzerland

23. Institute of Genetics and Genomics in Geneva (iGE3), University of Geneva, Geneva, Switzerland

24. Lausanne University Hospital, Lausanne, Switzerland

25. University of Helsinki, Helsinki, Finland

26. Garvan Institute of Medical Research, Garvan-Weizmann Centre for Cellular Genomics, Sydney, Australia

27. Singapore Immunology Network, Agency for Science, Technology and Research, Singapore, Singapore

28. Institute for Molecular Bioscience, University of Queensland, Brisbane, Australia

29. Leiden University Medical Center, Leiden, The Netherlands

30. Heart Center Leipzig, Universität Leipzig, Leipzig, Germany

31. Unit of Animal Genomics, WELBIO, GIGA-R \& Faculty of Veterinary Medicine, University of Liege, 1 Avenue de l'Hôpital, Liège 4000, Belgium

32. Wellcome Centre for Human Genetics, University of Oxford, Oxford OX3 7BN, United Kingdom

33. Department of Clinical Physiology, Tampere University Hospital and Faculty of Medicine and Life Sciences, University of Tampere, Tampere, Finland

34. Department of Computer Science, Johns Hopkins University, Baltimore, United States of America

35. Genetics and Genomic Science Department, Icahn School of Medicine at Mount Sinai, New York, United States of America

36. IFB Adiposity Diseases, Universität Leipzig, Leipzig, Germany

37. Interdisciplinary Center for Clinical Research, Faculty of Medicine, Universität Leipzig, Leipzig, Germany

38. Department of Medical Statistics and Bioinformatics, Leiden University Medical Center, Leiden, The Netherlands

39. Laboratory for Genotyping Development, RIKEN Center for Integrative Medical Sciences, Kanagawa 230-0045, Japan

40. DZHK (German Centre for Cardiovascular Research), partner site Munich Heart Alliance, Munich, Germany

41. Institute of Clinical Chemistry and Laboratory Medicine, Greifswald University Hospital, Greifswald, Germany

42. Faculty of Genes, Behavior and Health, Vrije Universiteit Amsterdam, Amsterdam, The Netherlands

43. Stanford University, Stanford, United States of America

44. Turku University Hospital and University of Turku, Turku, Finland

45. Department of Internal Medicine, Maastricht University Medical Centre, Maastricht, The Netherlands

46. Department of Medicine, Universität Leipzig, Leipzig, Germany

47. Department of Medical Epidemiology and Biostatistics, Karolinska Institutet, Stockholm, Sweden

48. Center for Molecular and Biomolecular Informatics, Radboud Institute for Molecular Life Sciences, Radboud University Medical Center Nijmegen, Nijmegen, The Netherlands

49. Institute for Laboratory Medicine, LIFE - Leipzig Research Center for Civilization Diseases, Universität Leipzig, Leipzig, Germany

50. University Medical Center Utrecht, Utrecht, The Netherlands

51. Interfaculty Institute for Genetics and Functional Genomics, University Medicine Greifswald, Greifswald, Germany

52. Institute for Molecular Bioscience, University of Queensland, Brisbane, Australia

53. Statistical and Translational Genetics, University of Helsinki, Helsinki, Finland

54. National Institute for Health and Welfare, University of Helsinki, Helsinki, Finland

55. Institute of Human Genetics, Helmholtz Zentrum München, Neuherberg, Germany

56. Institute of Human Genetics, Technical University Munich, Munich, Germany.

57. Kaiser Permanente Washington Health Research Institute, Seattle, WA, United States of America

58. Department of Medicine, University of Washington, Seattle, United States of America

59. Human Genetics, Wellcome Sanger Institute, Wellcome Genome Campus, Hinxton Cambridge, United Kingdom

60. Genome Biology Unit, European Molecular Biology Laboratory, 69117 Heidelberg, Germany

61. Division of Computational Genomics and Systems Genetics, German Cancer Research Center, 69120 Heidelberg, Germany

62. Departments of Biomedical Engineering and Computer Science, Johns Hopkins University, Baltimore, United States of America

63. Institute for Advanced Research, Wenzhou Medical University, Wenzhou, Zhejiang 325027, China

\# Correspondence can be addressed to

Urmo Võsa (urmo.vosa@gmail.com)

Annique Claringbould (anniqueclaringbould@gmail.com)

Lude Franke (ludefranke@gmail.com) 


\section{Summary}

While many disease-associated variants have been identified through genome-wide association studies, their downstream molecular consequences remain unclear.

To identify these effects, we performed cis- and trans-expression quantitative trait locus (eQTL) analysis in blood from 31,684 individuals through the eQTLGen Consortium.

We observed that cis-eQTLs can be detected for $88 \%$ of the studied genes, but that they have a different genetic architecture compared to disease-associated variants, limiting our ability to use cis-eQTLs to pinpoint causal genes within susceptibility loci.

In contrast, trans-eQTLs (detected for $37 \%$ of 10,317 studied trait-associated variants) were more informative. Multiple unlinked variants, associated to the same complex trait, often converged on trans-genes that are known to play central roles in disease etiology.

We observed the same when ascertaining the effect of polygenic scores calculated for 1,263 genome-wide association study (GWAS) traits. Expression levels of $13 \%$ of the studied genes correlated with polygenic scores, and many resulting genes are known to drive these traits. 


\section{Main text}

Expression quantitative trait loci (eQTLs) have become a common tool to interpret the regulatory mechanisms of the variants associated with complex traits through genome-wide association studies (GWAS). Cis-eQTLs, where gene expression levels are affected by a nearby single nucleotide polymorphism (SNP) ( $<1$ megabases; Mb), in particular, have been widely used for this purpose. However, cis-eQTLs from the genome tissue expression project (GTEx) explain only a modest proportion of disease heritability ${ }^{1}$.

In contrast, trans-eQTLs, where the SNP is located distal to the gene $(>5 \mathrm{Mb})$ or on other chromosomes, can provide insight into the effects of a single variant on many genes. Trans-eQTLs identified before ${ }^{1-7}$ have already been used to identify putative key driver genes that contribute to disease ${ }^{8}$. However, trans-eQTL effects are generally much weaker than those of cis-eQTLs, requiring a larger sample size for detection.

While trans-eQTLs are useful for the identification of the downstream effects of a single variant, a different approach is required to determine the combined consequences of trait-associated variants. Polygenic scores (PGS) have been recently applied to sum genome-wide risk for several diseases and likely will improve clinical care ${ }^{9,10}$. However, the exact consequences of different PGS at the molecular level, and thus the contexts in which a polygenic effects manifest themselves, are largely unknown. Here, we systematically investigate trans-eQTLs as well as associations between PGS and gene expression (expression quantitative trait score, eQTS) to determine how genetic effects influence and converge on genes and pathways that are important for complex traits.

To maximize the statistical power to detect eQTL and eQTS effects, we performed a large-scale meta-analysis in 31,684 blood samples from 37 cohorts (assayed using three gene expression 
platforms) in the context of the eQTLGen Consortium. This allowed us to identify significant ciseQTLs for 16,989 genes, trans-eQTLs for 6,298 genes and eQTS effects for 2,568 genes (Figure 1A), revealing complex regulatory effects of trait-associated variants. We combine these results with additional data layers and highlight a number of examples where we leverage this resource to infer novel biological insights into mechanisms of complex traits. We hypothesize that analyses identifying genes further downstream are more cell-type specific and more relevant for understanding disease (Figure 1B). 


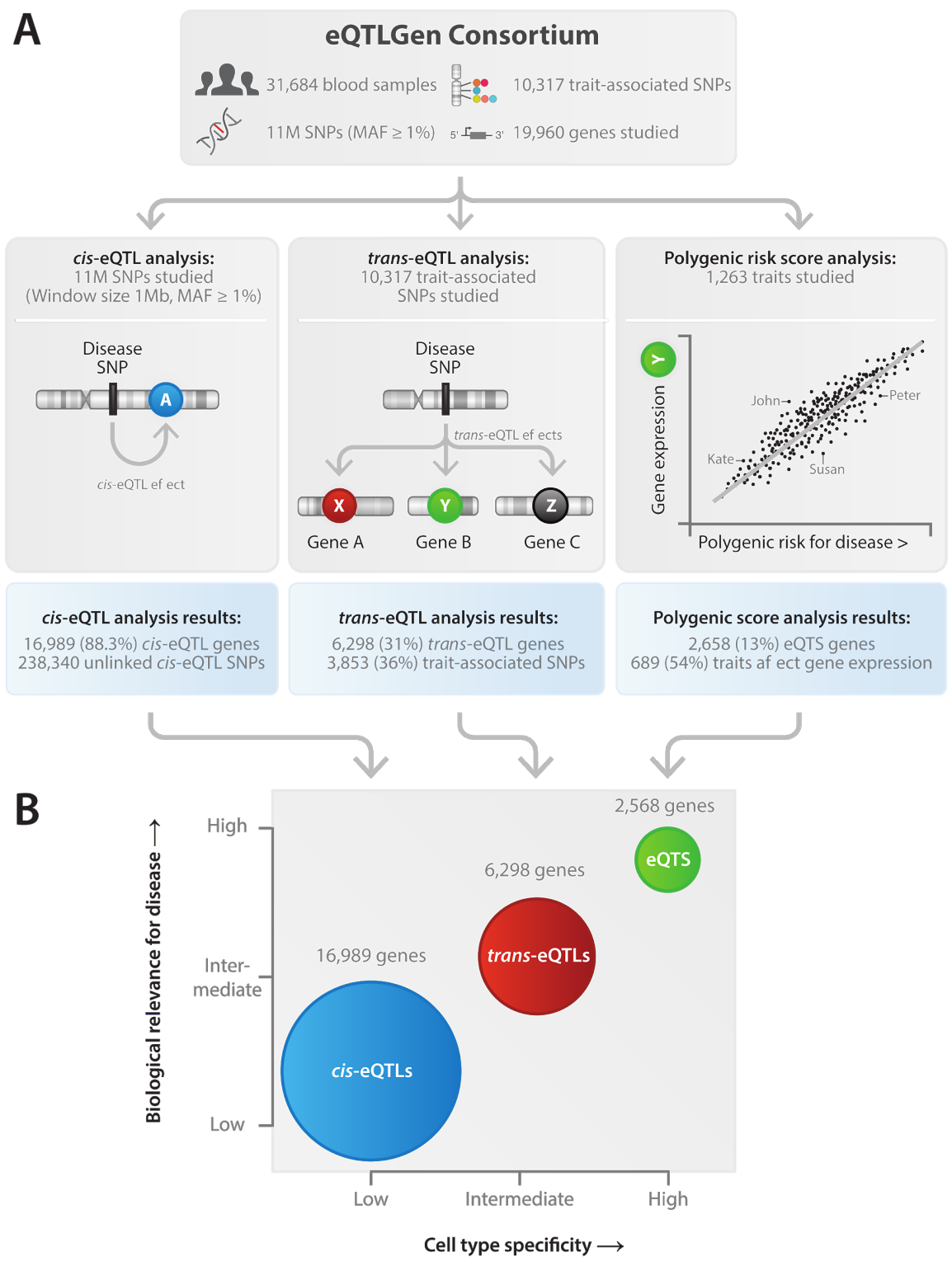

Figure 1. Overview of the study. (A) Overview of main analyses and their results. (B) Model of genetic effects on gene expression. Cis-eQTL are common and widely replicable in different tissues and cell types, whereas trans-eQTLs and eQTS are more cell type specific. The biological insight derived from our ciseQTL results are usually not well interpretable in the context of complex traits, suggesting that weaker distal effects give additional insight about biological mechanisms leading to complex traits. 


\section{Local genetic effects on gene expression in blood are widespread and}

\section{replicable in other tissues}

Using eQTLGen consortium data from 31,684 individuals, we performed cis-eQTL, trans-eQTL and eQTS meta-analyses (Figure 1A, Supplementary Table 1). Different expression profiling platforms were integrated using a data-driven method (Online Methods). To ensure the robustness of the identified eQTLs, we performed eQTL discovery per platform and replicated resulting eQTLs in the other platforms, observing excellent replication rates and consistency of allelic directions (Online Methods, Supplementary Note, Extended Data Figure 1A-C). We identified significant cis-eQTLs (SNP-gene distance $<1 \mathrm{Mb}$, gene-level False Discovery Rate (FDR) $<0.05$; Online Methods) for 16,989 unique genes $(88.3 \%$ of autosomal genes expressed in blood and tested in cis-eQTL analysis; Figure 1A). Out of 10,317 trait-associated SNPs tested, 1,568 (15.2\%) were in high linkage disequilibrium (LD) with the lead eQTL SNP showing the strongest association for a cis-eQTL gene, $\left(R^{2}>0.8\right.$; $1 \mathrm{kG}$ p1v3 EUR; Supplementary Table 2;

Online Methods). Genes highly expressed in blood but not under any detectable cis-eQTL effect were more likely $\left(P=2 \times 10^{-6}\right.$; Wilcoxon two-sided test; Figure $\left.2 A\right)$ to be intolerant to loss-of-function mutations in their coding region ${ }^{11}$, suggesting that eQTLs on such gene would interfere with the normal functioning of the organism.

We observed that $92 \%$ of the lead cis-eQTL SNPs map within $100 \mathrm{~kb}$ of the gene (Figure 2D), and this increased to $97.2 \%$ when only looking at the $20 \%$ of the genes with the strongest lead ciseQTL effects. Of these strong cis-eQTLs, $84.1 \%$ of the lead eQTL SNPs map within $20 \mathrm{~kb}$ of the gene. GWAS simulations ${ }^{12}$ indicate that lead GWAS signals map within $33.5 \mathrm{~kb}$ from the causal variant in $80 \%$ of cases, which suggests that our top SNPs usually tag causal variants that map 
directly into either the promoter region, the transcription start site (TSS), the gene body, or the transcription end site (TES). For strong cis-eQTLs we observed that lead cis-eQTL SNPs located $>100 \mathrm{~kb}$ from the TSS or TES overlap capture Hi-C contacts (37\%; Figure 2E) more often than short-range cis-eQTL effects $\left(16 \%\right.$; Chi $^{2}$ test $\left.P=2 \times 10^{-5}\right)$, indicating that, for long-range cis-eQTLs the SNP and gene often physically interact to cause the cis-eQTL effect. For instance, a capture Hi-C contact for IRS1 overlapped the lead eQTL SNP, mapping 630kb downstream from IRS1 (Figure 2F).

We observed that our sample-size improved fine-mapping: for 5,440 protein-coding cis-eQTL genes that we had previously identified in 5,311 samples $^{1}$ we now observe that the lead SNP typically map closer to the cis-eQTL gene (Extended Data Figure 4).

Cis-eQTLs showed directional consistency across tissues: in 47 postmortem tissues (GTEx $v 7^{13}$ ) we observed an average of $14.8 \%$ replication rate (replication FDR<0.05 in GTEx; median 15.1\%; range 3.6-29.7\%; whole blood tissue excluded) and on average a $95.0 \%$ concordance in allelic directions (median 95.3\%, range 86.7-99.3\%; whole blood tissue excluded) among the cis-eQTLs that significantly replicated in GTEx (Extended Data Figure 5, Supplementary Note and Supplementary Table 3).

However, our lead cis-eQTL SNPs show significantly different epigenetic histone mark characteristics, as compared to 3,668 SNPs identified in GWAS (and associated to blood related traits or immune-mediated diseases to minimize potential confounding). We observed significant differences for 20 out of 32 tested histone marks with H3K36me3, H3K27me3, H3K79me1 and H2BK20ac showing the strongest difference (Wilcoxon $P=10^{-39}, 10^{-21}, 10^{-19}$ and $10^{-18}$, 
respectively), suggesting that cis-eQTLs have a different genetic architecture, as compared to complex traits and diseases.

We tested this for 16 well-powered complex traits (Supplementary Table 20) and observed that genes prioritized by combining cis-eQTL and GWAS data using summary statistics based Mendelian randomization (SMR ${ }^{14}$; Online Methods) did not overlap significantly more with genes prioritized through an alternative method (DEPICT) that does not use any cis-eQTL information ${ }^{15}$. While the genes prioritized with SMR were informative, and enriched for relevant pathways for several immune traits (Supplementary Table 20), non-blood-trait-prioritized genes were difficult to interpret in the context of disease. Moreover, the lack of enriched overlap between DEPICT and SMR indicates that employing cis-eQTL information does not necessarily clarify which genes are causal for a given susceptibility locus. As such, some caution is warranted when using a single ciseQTL repository for interpretation of GWAS.

\section{One third of trait-associated variants have trans-eQTL effects}

An alternative strategy for gaining insight into the molecular functional consequences of diseaseassociated genetic variants is to ascertain trans-eQTL effects. We tested 10,317 trait-associated SNPs ( $\mathrm{P} \leq 5 \times 10^{-8}$; Online Methods, Supplementary Table 2) for trans-eQTL effects (SNP-gene distance $>5 \mathrm{Mb}$, FDR $<0.05$ ) to better understand their downstream consequences. We identified a total of 59,786 significant trans-eQTLs (FDR<0.05; Supplementary Table 4, Extended Data Figure 6), representing 3,853 unique SNPs (37\% of tested GWAS SNPs) and 6,298 unique genes (32\% of tested genes; Figure 1A). When compared to the previous largest trans-eQTL metaanalysis $^{1}(\mathrm{~N}=5,311 ; 8 \%$ of trait-associated SNPs with a significant trans-eQTL), these results 
indicate that a large sample size is critical for identifying downstream effects. Colocalization analyses in a subset of samples ( $n=4,339$; Supplementary Note) using COLOC ${ }^{16}$ estimated that $52 \%$ of trans-eQTL signals colocalize with at least one cis-eQTL signal (posterior probability > 0.8 ;

Extended Data Figure 7A-B). Corresponding colocalizing cis-eQTL genes were enriched for transcription factor activity ("regulation of transcription from RNA polymerase II promoter"; P < $1.3 \times 10^{-9} ;$ Extended Data Figure 7C). Finally, highly expressed genes without a detectable transeQTL effect were more likely to be intolerant to loss-of-function variants $\left(P=6.4 \times 10^{-7}\right.$; Wilcoxon test, Figure 2B), similar to what we observed for cis-eQTLs. 
A B

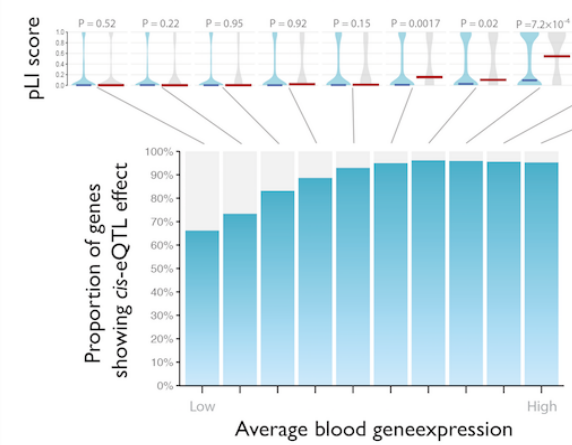

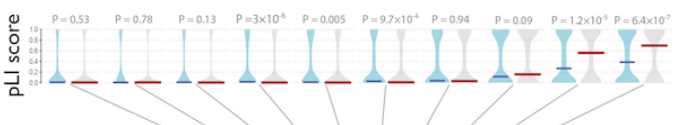

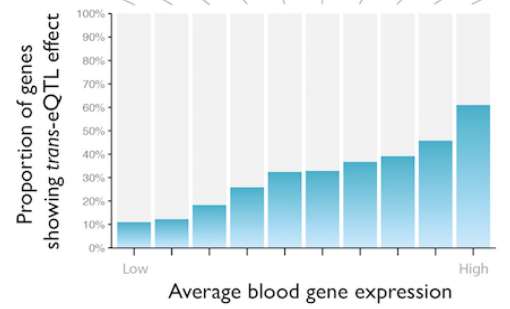

C
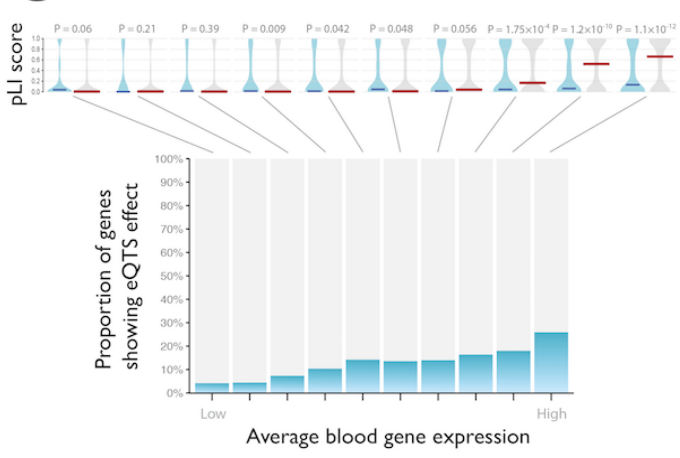

D

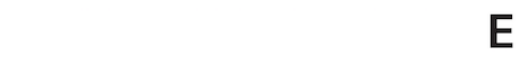

genes cis-eQTLenes

E
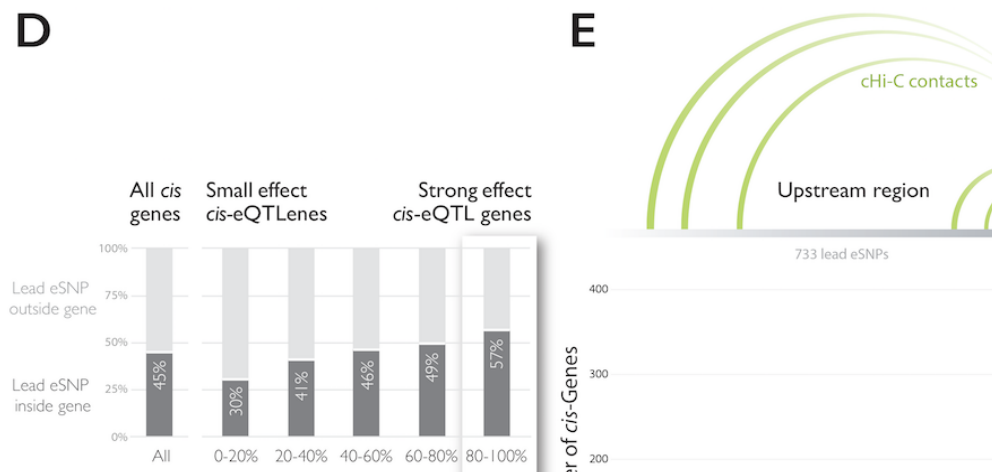

Proportion

of lead eSNPs of lead eSNPs of lead eSNPS

Dokb of gene $<50 \mathrm{~kb}$ of gene $20 \mathrm{kh}$ of gere

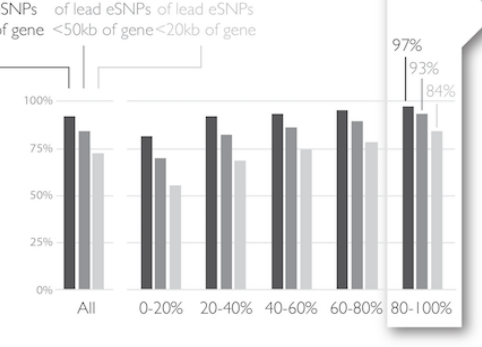

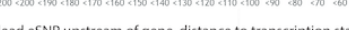

Capture Hi-C contacts:

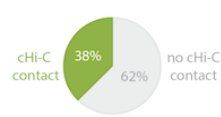

Lead eSNP > 100kb from TSS
Enrichment of epigenetic mark at cis-eQTL SNP locations compared to trait-associated SNP locations
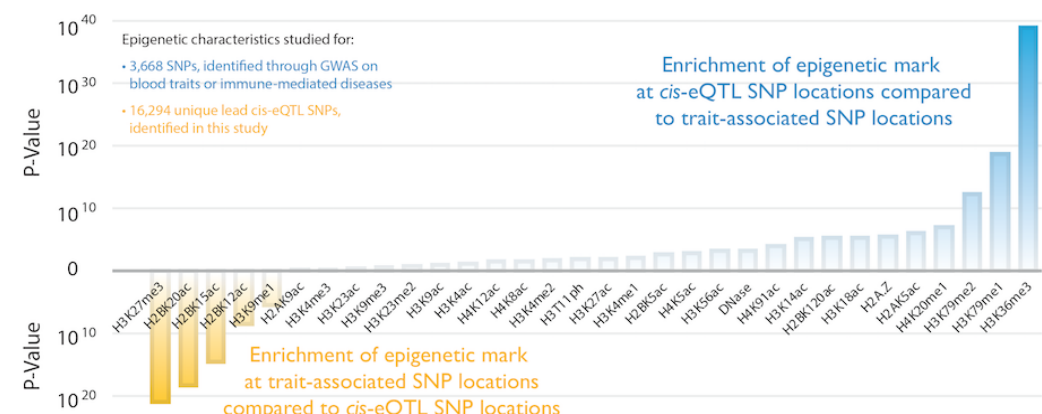
Figure 2. Results of the cis- and trans-eQTL analysis. All genes tested in (A) cis-eQTL analysis, (B) trans-eQTL analysis, and (C) eQTS analysis were divided into 10 bins based on average expression levels of the genes in blood. Highly expressed genes without any eQTL effect (grey bars) were less tolerant to loss-of-function variants (Wilcoxon test on pLI scores). Indicated are medians per bin. (D) Genes with strong effect sizes are more likely to have a lead SNP within (top panel) or close to the gene (bottom panel) (E) Top cis-eQTL SNPs positioning further from transcription start site (TSS) and transcription end site (TES) are more likely to overlap capture Hi-C contacts with TSS. (F) Enrichment analyses on epigenetic marks of cis-eQTL lead SNPs, compared to SNPs identified through GWAS and associated to blood-related or immune-mediated diseases, reveal significant differences in epigenetic characteristics.

In order to study the biological nature of the trans-eQTLs we identified, we conducted several enrichment analyses (Supplementary Note, Extended Data Figure 8, Figure 3). We observed 2.2 fold enrichment for known transcription factor $(\mathrm{TF})$ - target gene pairs ${ }^{17}$ (Fisher's exact test $\mathrm{P}=$ 10-62; Supplementary Note), with the fold enrichment increasing to 3.2 (Fisher's exact test $P<10^{-}$ ${ }^{300}$ ) when co-expressed genes were included to TF targets. Those genes are potentially further downstream of respective TF targets in the molecular network. Similarly, we observed 1.19 fold enrichment of protein-protein interactions ${ }^{18}$ among trans-eQTL gene-gene pairs (Fisher's exact test $\mathrm{P}=0.05)$. Some of these cis-trans gene pairs encode subunits of the same protein complex (e.g. POLR3H and POLR1C). While significant cis-trans gene pairs were enriched for gene pairs showing co-expression (Pearson $R>0.4$; Fisher's exact test $P=10^{-35}$ ), we did not observe any enrichment of chromatin-chromatin contacts ${ }^{19}$ (0.99 fold enrichment; Fisher's exact test $\left.P=0.3\right)$. Using the subset of 3,831 samples from BIOS, we also ascertained whether the trans-eQTL effect was mediated through a gene that mapped within $100 \mathrm{~kb}$ from the trans-eSNP (i.e. using the cisgene as $\mathrm{G} \times \mathrm{E}$ term). We observed significant interaction effects for 523 SNP-cis-trans-gene 
combinations (FDR < 0.05; Supplementary Table 5), reflecting a 5.3 fold enrichment compared to what is expected by chance (Fisher's exact $P=7 \times 10^{-67}$ ). For instance, for rs7045087 (associated to red blood cell counts) we observed that the expression of interferon gene DDX58 (mapping 38bp downstream from rs7045087) significantly interacted with trans-eQTL effects on interferon genes HERC5, OAS1, OAS3, MX1, IFIT1, IFIT2, IFIT5, IFI44, IFI44L, RSAD2 and SAMD9 (Extended Data Figure 9). 


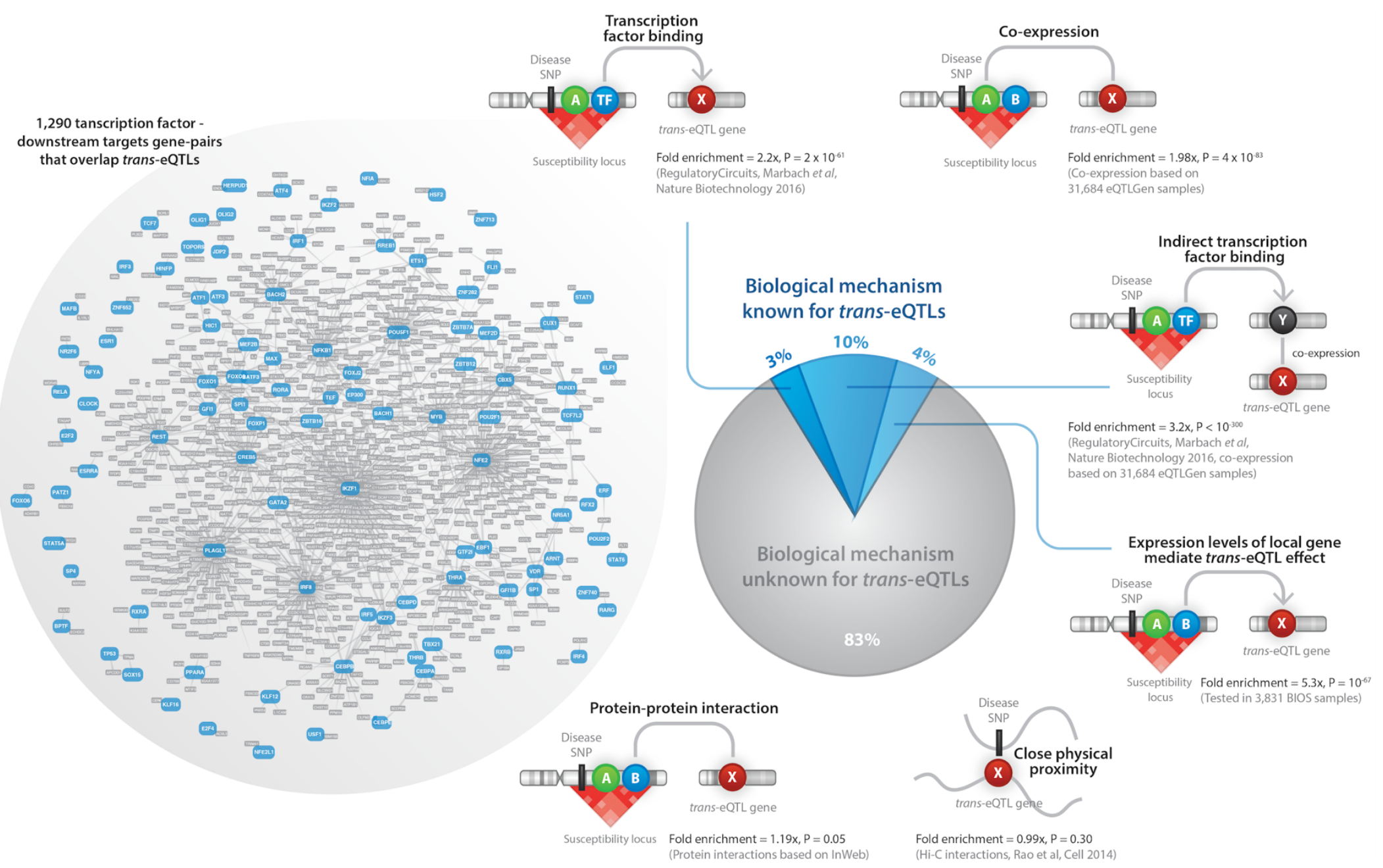

Figure 3. Mechanisms leading to trans-eQTLs. Shown are the results of enrichment analyses for known TF associations, HiC contacts, protein-protein interactions, gene co-expression and mediation analyses.

We estimate that $17.4 \%$ of the identified trans-eQTLs are explainable by (indirect) TF binding or mediation by cis-genes (Supplementary Note). This leaves $82.6 \%$ of the observed trans-eQTL effects unexplained. While it is likely that many of these trans-eQTLs reflect unknown (indirect) effects of TFs, we speculate that novel and unknown regulatory mechanisms could also play a role. By making all trans-eQTL results (irrespective of their statistical significance) publicly available, we envision this dataset will help to yield such insight in the future. 
To estimate the proportion of loci where the trait-associated SNP explained the trans-eQTL signal in the locus, we performed locus-wide conditional trans-eQTL analysis in a subset of 4,339 samples for 12,991 trans-eQTL loci (Online Methods; Extended Data Figure 10; Supplementary Table 6). In $43 \%$ of these loci, we observed that the trait-associated SNP was in high LD with the transeQTL SNP having the strongest association in the locus $\left(R^{2}>0.8,1 \mathrm{kG}\right.$ p1v3 EUR; Supplementary Table 7). For 95 cases, the strongest cis- and trans-eQTL SNPs were both in high LD with GWAS SNP ( $R^{2}>0.8$ between top SNPs, 1kG p1v3 EUR; Supplementary Table 7).

The majority (64\%) of trans-eQTL SNPs have previously been associated with blood composition phenotypes, such as platelet count, white blood cell count and mean corpuscular volume ${ }^{20}$. In comparison, blood cell composition SNPs from the same study comprised only $20.7 \%$ od all the tested trait-associated SNPs. This was expected, since SNPs that regulate the abundance of a specific blood cell type would result in trans-eQTL effects on genes, specifically expressed in that cell type.

Therefore, we aimed to distinguish trans-eQTLs caused by intracellular molecular mechanisms from blood cell type QTLs using eQTL data from lymphoblastoid cell line (LCL), induced pluripotent cells (iPSCs), several purified blood cell types (CD4+, CD8+, CD14+, CD15+, CD19+, monocytes and platelets) and blood DNA methylation QTL data. In total, 3,853 (6.4\%) of trans-eQTLs showed significant replication in at least one cell type or in the methylation data (Extended Data Figure 11, Supplementary Table 11A). While this set of trans-eQTLs (denoted as the "intracellular eQTLs") is less likely to be driven by cell type composition, we acknowledge that the limited sample size of the available trans-eQTL replication datasets make our replication effort very conservative. Furthermore, trans-eQTLs caused by variants associated with cell type proportions may be 
informative for understanding the biology of a trait. Therefore, we did not remove these kinds of trans-eQTLs from our interpretative analyses.

Next, we aimed to replicate the identified trans-eQTLs in the tissues from GTEx ${ }^{13}$. Although the replication rate was very low $(0-0.03 \%$ of trans-eQTLs replicated in non-blood tissues, FDR $<0.05$, same allelic direction; Supplementary Table 11B), we did observe an inflation of signal (median chi-squared statistic) for identified trans-eQTLs in several GTEx tissues (Extended Data Figure 12). Non-blood tissues showing the strongest inflation were liver, heart atrial appendage and nonsun-exposed skin.

\section{Trans-eQTLs are effective for discerning the genetic basis of complex traits}

As described above, trans-eQTLs can arise due to cis-eQTL effects on TFs, whose target genes show trans-eQTL effects. We describe below such examples, but also highlight trans-eQTLs where the eQTL SNP works through a different mechanism.

Combining cis- and trans-eQTL effects can pinpoint the genes acting as drivers of transeQTL effects. For example, the age-of-menarche-associated SNP rs $1532331^{21}$ is in high LD with the top cis-eQTL effect for transcription factor ZNF131 ( $\mathrm{R}^{2}>0.8,1 \mathrm{kG}$ p1v3 EUR). Cis-eQTL and trans-eQTL effects for this locus co-localized for 25 out of the 75 downstream genes (Figure 4A). In a recent short hairpin RNA knockdown experiment of ZNF13122, three separate cell isolates showed downregulation of four genes that we identified as trans-eQTL genes: HAUS5, TMEM237, MIF4GD and AASDH (Figure 4A). ZNF131 has been hypothesized to inhibit estrogen signaling ${ }^{23}$, which may explain how the SNP in this locus contributes to altering the age of menarche. 
bioRxiv preprint doi: https://doi.org/10.1101/447367; this version posted October 19, 2018. The copyright holder for this preprint (which was not certified by peer review) is the author/funder. All rights reserved. No reuse allowed without permission.

A

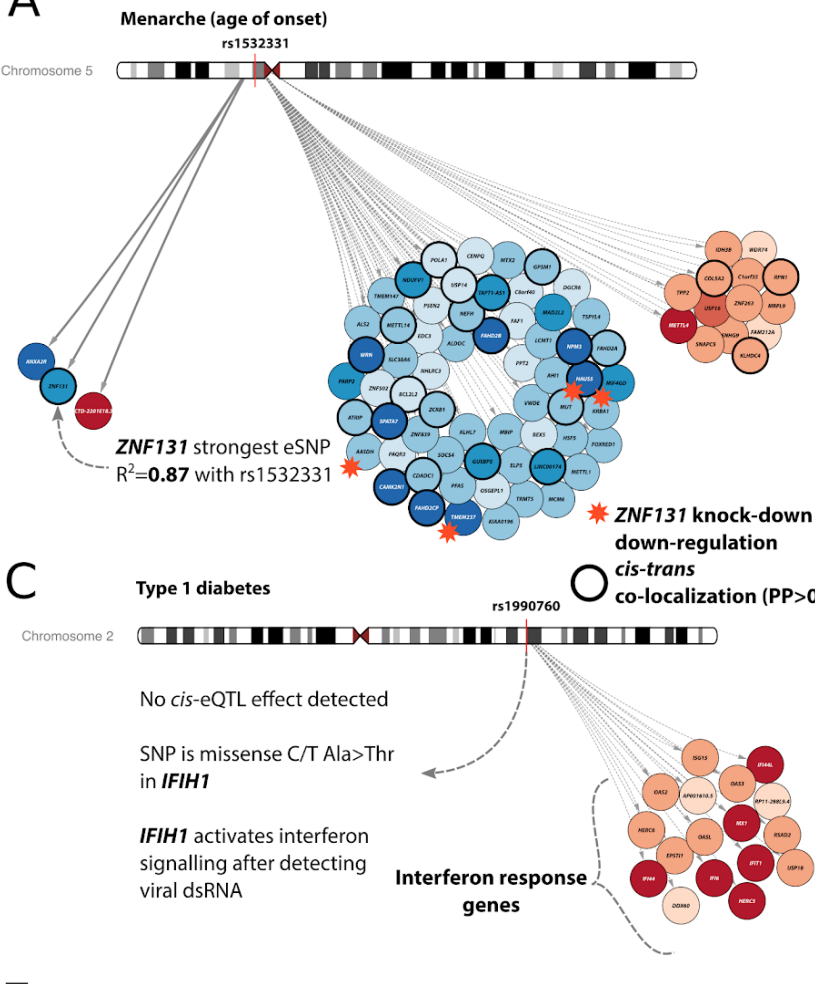

\section{E}

Asthma

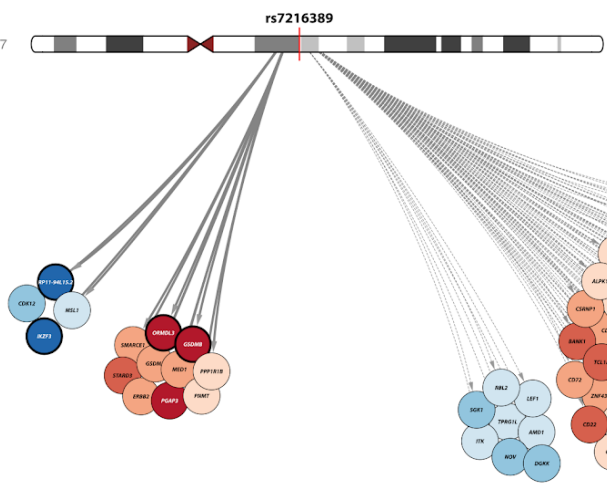

F

Coronary artery disease

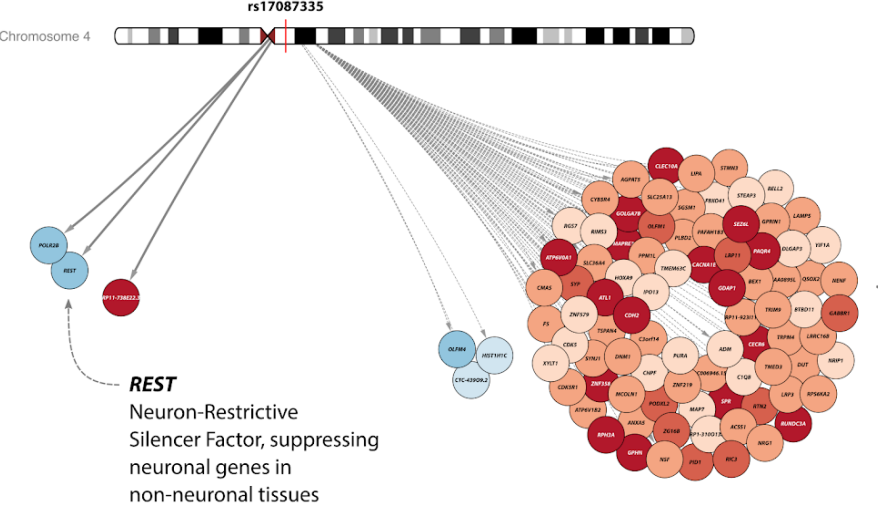

neuronal genes in

eQTL effect size

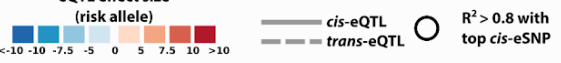

B Phospholipid levels

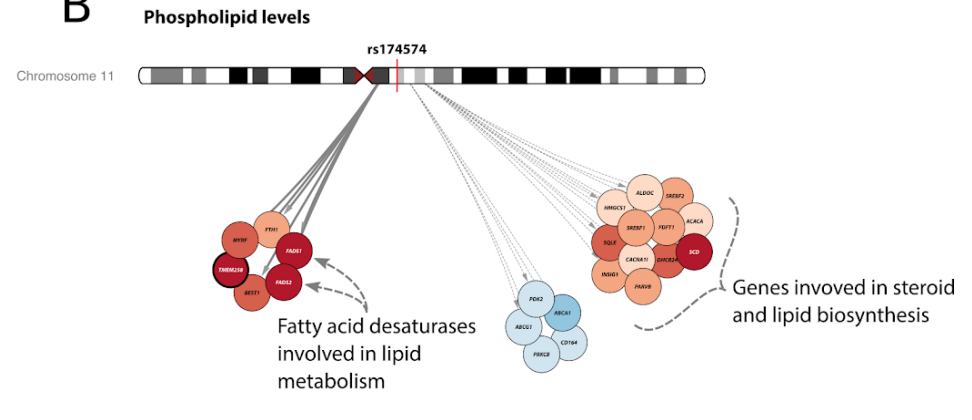

D

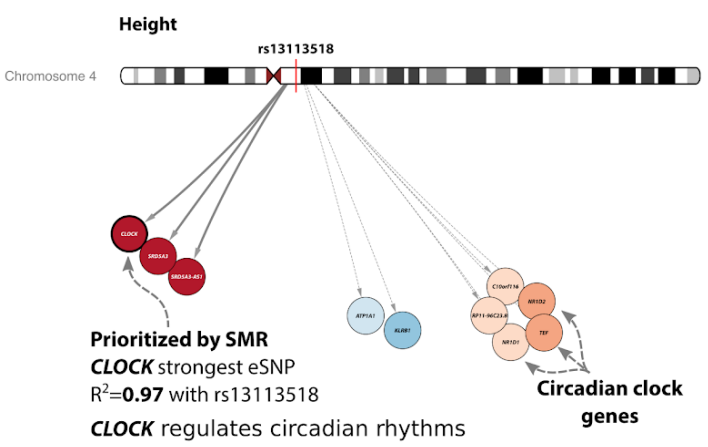

CLOCK regulates circadian rhythms genes
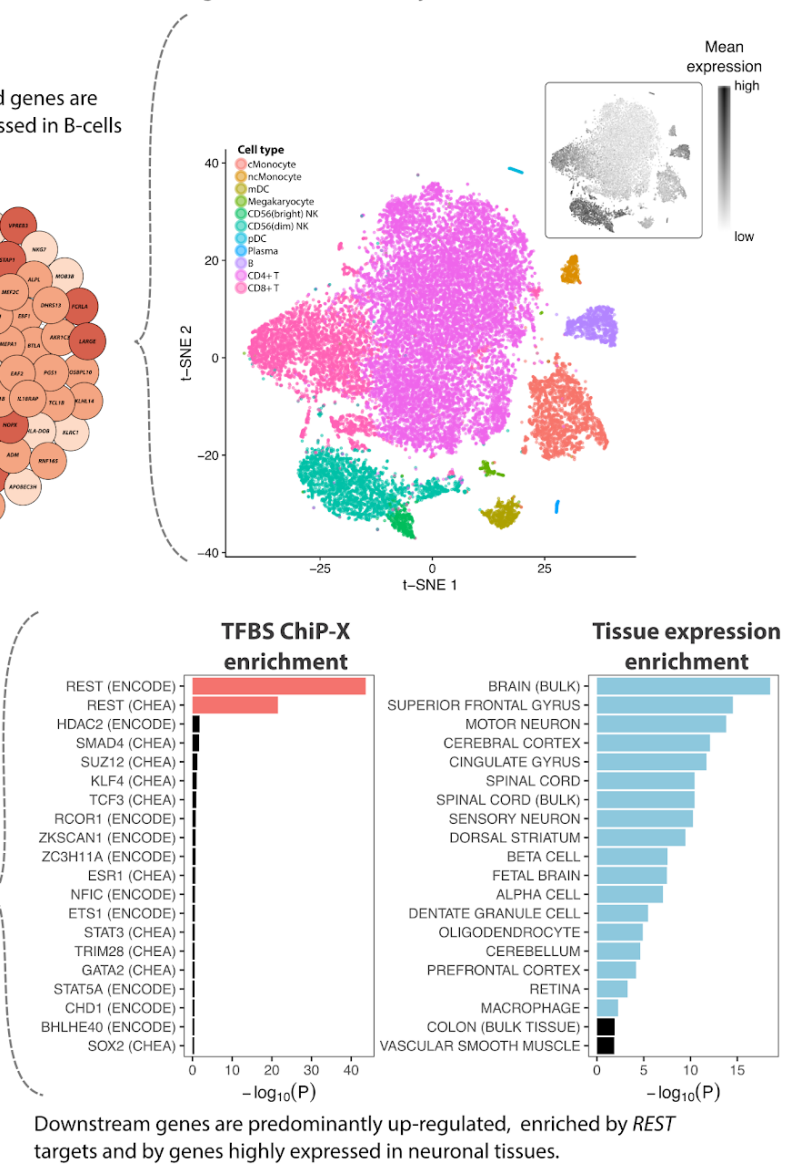

Downstream genes are predominantly up-regulated, enriched by REST 
Figure 4. Examples of cis- and trans-eQTLs. (A) Cis-eQTL on ZNF131 is prioritized because several trans-eQTL genes are down-regulated by ZNF131 in functional study. (B) Phospholipidassociated SNP shows cis-and trans-eQTLs on lipid metabolism genes. (C) Type I diabetes associated SNP has no cis-eQTLs, but trans-eQTL genes point to interferon signaling pathway. (D) Circadian rhythm genes CLOCK (in cis) and NR1D1, NR1D2, TEF (in trans) identified for height associated SNP. (E) eQTLs for asthma SNP tag cell type abundance of B and NK cells. (F) TranseQTL genes for REST locus are highly enriched for REST transcription factor targets and for neuronal expression.

Trans-eQTLs extend insight for loci with multiple cis-eQTL effects. In the FADS1/FADS2 locus, rs174574 is associated with lipid levels ${ }^{24}$ and affects 17 genes in trans (Figure 4B). The strongest cis-eQTLs modulate the expression of FADS1, FADS2 and TMEM258, with latter being in high LD with GWAS SNP ( $\mathrm{R}^{2}>0.8,1 \mathrm{kG}$ p1v3 EUR). FADS1 and FADS2 have been implicated ${ }^{24}$ since they regulate fatty acid synthesis, and consistent with their function, trans-eQTL genes from this locus are highly enriched for triglyceride metabolism $\left(P<4.1 \times 10^{-9}\right.$, GeneNetwork ${ }^{25}$ REACTOME pathway enrichment). Since this locus has extensive LD, variant and gene prioritization is difficult: conditional analyses in 4,339 sample subset showed that each of cis-eQTL gene is influenced by more than one SNP, but none of these are in high LD with rs $174574\left(R^{2}<\right.$ 0.8, 1 kG p1v3, EUR). As such, our trans-eQTL analysis results are informative for implicating FADS1 and FADS2, whereas cis-eQTLs are not.

Trans-eQTLs can shed light on loci with no detectable cis-eQTLs. rs1990760 is associated with multiple immune-related traits (Type 1 Diabetes (T1D), Inflammatory bowel disease (IBD), Systemic Lupus Erythematosis (SLE) and psoriasis ${ }^{26-29}$ ). For this SNP we identified 17 trans-eQTL effects, but no detectable gene-level cis-eQTLs in blood (Figure 4C) and GTEx. However, the risk 
allele for this SNP causes an Ala946Thr amino acid change in the RIG-1 regulatory domain of MDA5 (encoded by IFIH1 - Interferon Induced With Helicase C Domain 1), outlining one possible mechanism leading to the observed trans-eQTLs. MDA5 acts as a sensor for viral double-stranded RNA, activating interferon I signalling among other antiviral responses. All the trans-eQTL genes were up-regulated relative to risk allele to T1D, and $9(52 \%)$ are known to be involved in interferon signaling (Supplementary Table 12).

Trans-eQTLs can reveal cell type composition effects of the trait-associated SNP. TranseQTL effects can also show up as a consequence of a SNP that alters cell-type composition. For example, the asthma-associated SNP rs7216389 30 has 14 cis-eQTL effects, most notably on IKZF3, GSDMB, and ORMDL3 (Figure 4E). SMR prioritized all three cis-genes equally (Extended Data Figure 13), making it difficult to draw biological conclusions (similar as we observed for the FADS locus). However, 94 out of the 104 trans-eQTL genes were up-regulated by the risk allele for rs7216389 and were mostly expressed in B cells and natural killer cells ${ }^{31}$ (Figure 4E). IKZF3 is part of the Ikaros transcription factor family that regulates B-cell proliferation ${ }^{31,32}$, suggesting that a decrease of IKZF3 leads to an increased number of B cells and concurrent trans-eQTL effects caused by cell-type composition differences.

Some trans-eQTLs influence genes strongly expressed in tissues other than blood. We observed trans-eQTL effects on genes that are hardly expressed in blood, indicating that our transeQTL effects are informative for non-blood related traits as well: rs17087335, which is associated with coronary artery disease ${ }^{33}$, affects the expression of 88 genes in trans (Figure $4 \mathbf{F}$ ), that are highly expressed in brain (hypergeometric test, ARCHS4 database, q-value $=2.58 \times 10^{-17}$; Figure 4F, Supplementary Table 13), but show very low expression in blood. SNPs linked with rs17087335 $\left(R^{2}>0.8,1 \mathrm{kG}\right.$ p1v3 EUR) are associated with height (rs2227901, rs3733309 and 
rs 17081935$)^{34,35}$, and platelet count $(\text { rs } 7665147)^{20}$. The minor alleles of these SNPs downregulate the nearby gene REST (RE-1 silencing transcription factor), although none of these variants is in LD $\left(\mathrm{R}^{2}<0.2\right.$, 1kG p1v3 EUR) with the lead cis-eQTL SNP for REST. REST is a TF that downregulates the expression of neuronal genes in non-neuronal tissues ${ }^{36,37}$. It also regulates the differentiation of vascular smooth muscles, and is thereby associated with coronary phenotypes ${ }^{38}$. 85 out of $88(96.6 \%)$ of the trans-eQTL genes were upregulated relative to the minor allele and were strongly enriched by transcription factor targets of REST (hypergeometric test for ENCODE REST ChIP-seq, q-value $=1.36 \times 10^{-42}$, Figure 4F). As such, trans-eQTL effects on neuronal genes implicate REST as the causal gene in this locus.

Trans-eQTLs identify pathways not previously associated with a phenotype. Some transeQTLs suggest the involvement of pathways which are not previously thought to play a role for certain complex traits: SMR analysis prioritized CLOCK as a potential causal gene in the heightassociated locus on chr 4q12 ( $P_{S M R}=3 \times 10^{-25}$; $P_{\text {HEIDI }}=0.02$; Figure 4D). In line with that, heightassociated SNP rs13113518 34 is also in high LD ( $\mathrm{R}^{2}>0.8,1 \mathrm{kG}$ p1v3 EUR) with the top cis-eQTL SNP for CLOCK. The upregulated TF CLOCK forms a heterodimer with TF BMAL1, and the resulting protein complex regulates circadian rhythm ${ }^{39}$. Three known circadian rhythm trans-eQTL genes (TEF, NR1D1 and NR1D2) showed increased expression for the trait-increasing allele, suggesting a possible mechanism for the observed trans-eQTLs through binding of CLOCK:BMAL1. TEF is a D-box binding TF whose gene expression in liver and kidney is dependent on the core circadian oscillator and it regulates amino acid metabolism, fatty acid metabolism and xenobiotic detoxification (Gachon et al., 2006). NR1D1 and NR1D2 encode the transcriptional repressors Rev-ErbA alpha and beta, respectively, and form a negative feedback loop to suppress BMAL1 expression ${ }^{40}$. NR1D1 and NR1D2 have been reported to be associated 
with osteoblast and osteoclast functions ${ }^{41}$, revealing a possible link between circadian clock genes and height.

Unlinked trait-associated SNPs converge on the same downstream genes in trans. We subsequently ascertained, per trait, whether unlinked trait-associated variants showed trans-eQTL effects on the same downstream gene. Here we observed 47 different traits where at least four independent variants affected the same gene in trans, $3.4 \times$ higher than expected by chance $(P=$ 0.001; two-tailed two-sample test of equal proportions; Supplementary Table 8). For SLE, for example, we observed that the gene expression levels of IFI44L, HERC5, IFI6, IFI44, RSAD2, MX1, ISG15, ANKRD55, OAS3, OAS2, OASL and EPSTI1 (nearly all interferon genes) were affected by at least three SLE-associated genetic variants, clearly showing the involvement of interferon signaling in SLE (Figure 5). 


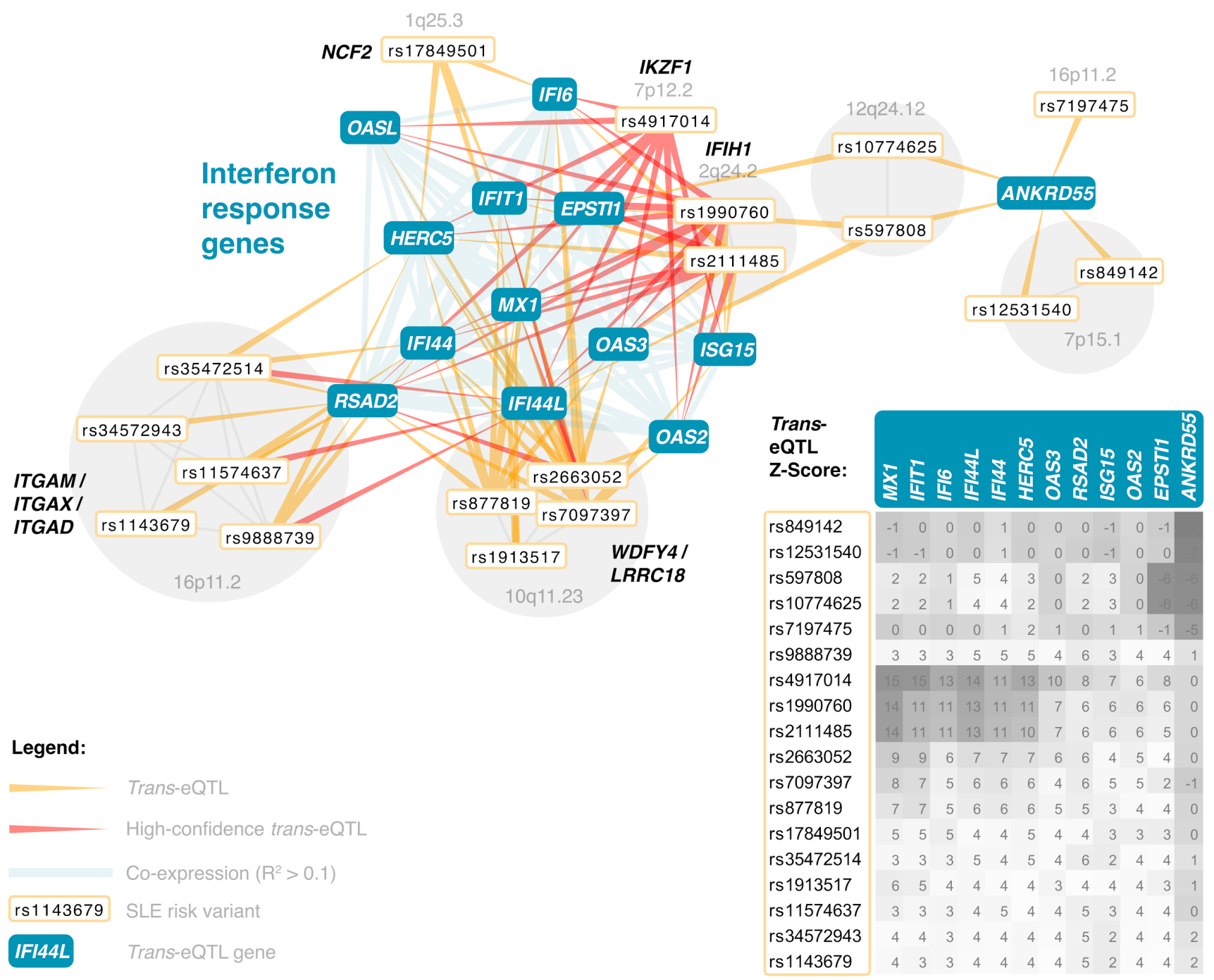

Figure 5. SNPs associated with SLE converge to the shared cluster of interferon response genes. Shown are genes which are affected by at least three independent GWAS SNPs. SNPs in partial LD are grouped together. Heat map indicates the direction and strength of individual trans-eQTL effects (Z-scores).

This convergence of multiple SNPs on the same genes lends credence to recent hypotheses with regards to the 'omnigenic' architecture of complex traits ${ }^{8}$ : indeed multiple unlinked variants do affect the same 'core' genes. The recent omnigenic model ${ }^{42}$ proposes a strategy to partition between core genes, which have direct effects on a disease, and peripheral genes, which can only affect disease risk indirectly through regulation of core genes. In Supplementary Equations, we 
show that this model also implies a correlation between polygenic risk scores and expression of core genes. We therefore studied this systematically by aggregating multiple associated variants into polygenic scores and ascertaining how they correlate with gene expression levels.

\section{eQTSs identify key driver genes for polygenic traits}

To ascertain the coordinated effects of trait-associated variants on gene expression, we used available GWAS summary statistics to calculate PGSs for 1,263 traits in 28,158 samples (Online Methods, Supplementary Table 14). We reasoned that when a gene shows expression levels that significantly correlate with the PGS for a specific trait (an expression quantitative trait score; eQTS), the downstream trans-eQTL effects of the individual risk variants converge on that gene, and hence, that the gene may be a driver of the disease.

Our meta-analysis identified 18,210 eQTS effects (FDR < 0.05), representing 689 unique traits (54\%) and 2,568 unique genes (13\%; Supplementary Table 15, Figure 1A). As expected, most eQTS associations represent blood cell traits (Extended Data Figure 14, Supplementary Table 16): for instance the PGS for mean corpuscular volume correlated positively with the expression levels of genes specifically expressed in erythrocytes, such as genes coding for hemoglobin subunits. However, we also identified eQTS associations for genes that are known drivers of other traits.

For example, 11 out of 26 genes associating with the PGS for high density lipoprotein levels (HDL ${ }^{43,44}$; FDR<0.05; Figure 6A) have previously been associated with lipid or cholesterol metabolism (Supplementary Table 18). $A B C A 1$ and $A B C G 1$, which positively correlated with the PGS for high HDL, mediate the efflux of cholesterol from macrophage foam cells and participate in 
HDL formation. In macrophages, the downregulation of both $A B C A 1$ and $A B C G 1$ reduced reverse cholesterol transport into the liver by $\mathrm{HDL}^{45}$ (Figure 6B). The genetic risk for high HDL was also negatively correlated with the expression of the low density lipoprotein receptor $L D L R$ (strongest eQTS $P=3.35 \times 10^{-20}$ ) known to cause hypercholesterolemia ${ }^{46}$. Similarly, the gene encoding the TF SREBP-2, which is known to increase the expression of $L D L R$, was downregulated (strongest eQTS $\left.P=3.08 \times 10^{-7}\right)$. The negative correlation between SREBF2 expression and measured HDL levels has been described before ${ }^{47}$, indicating that the eQTS reflects an association with the actual phenotype. Zhernakova et al. proposed a model where down-regulation of SREBF2 results in the effect on its target gene FADS2. We did not observe a significant HDL eQTS effect on FADS2 (all eQTS P>0.07), possibly because the indirect effect is too small to detect. We hypothesize that HDL levels in blood can result in a stronger reverse cholesterol transport into the liver, which may result in downregulation of $L D L R^{48}$ 

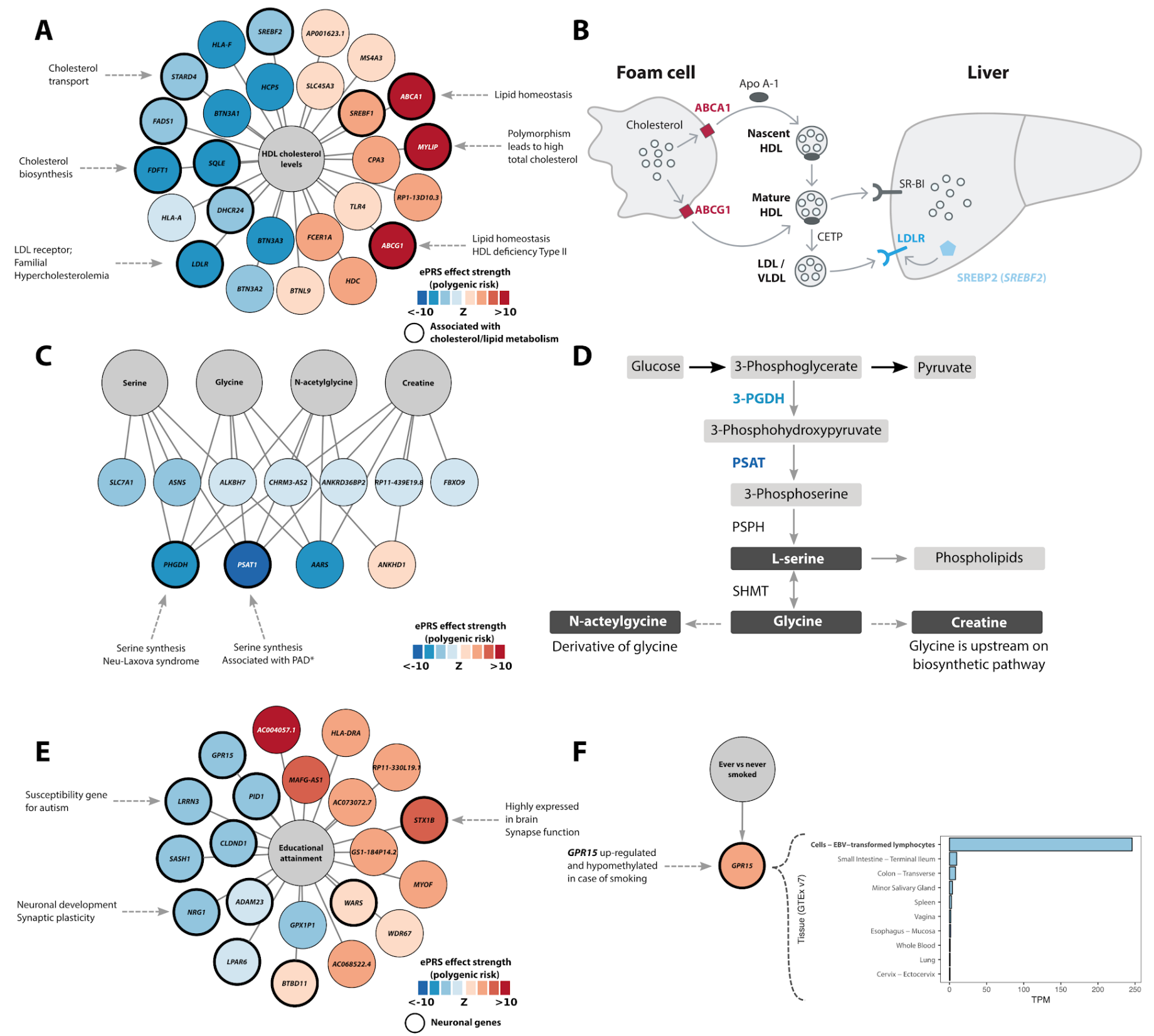

$\mathbf{G}$

Ulcerative Colitis

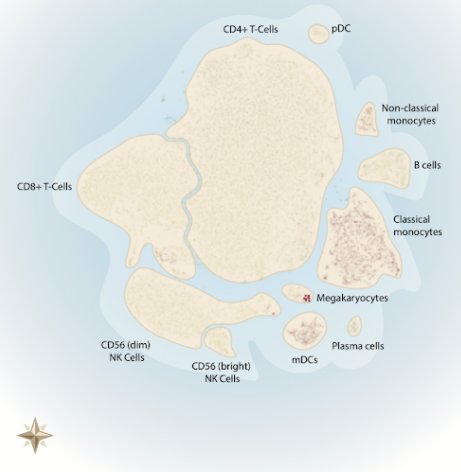

Systemic Lupus Erythematosis
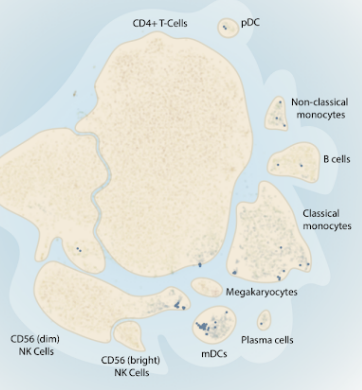

Celiac disease

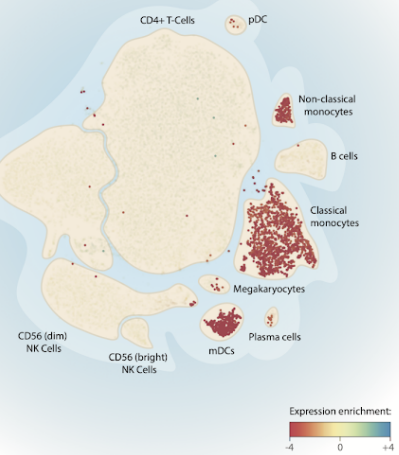


Figure 6. Examples of eQTS. (A) Polygenic risk score (PRS) for high density lipoprotein associates to lipid metabolism genes. (B) The role of $A B C A 1, A B C G 1$, and SREBF2 in cholesterol transport. (C) Polygenic scores for serine, glycine, n-acetylglycine and creatine levels negatively associate with gene expression of PHGDH, PSAT1, and AARS. (D) Serine biosynthesis pathway. (E) PRS for educational attainment identifies genes with neuronal functions. (F) Polygenic score for smoking status upregulates GPR15, which plays a role in lymphocyte differentiation. (G) eQTS genes for immune-related diseases are enriched for genes specifically expressed in certain blood cell types.

eQTS analysis also identified genes relevant for non-blood traits, such as the association of GPR15 $\left(P=3.7 \times 10^{-8}, F D R<0.05\right.$; Figure $\left.6 F\right)$ with the trait 'ever versus never smoking' 49. GPR15 is a biomarker for smoking ${ }^{50}$ that is overexpressed and hypomethylated in smokers ${ }^{51}$. We observe strong GPR15 expression in lymphocytes (Figure 6F), suggesting that the association with smoking could originate from a change in the proportion of T cells in blood ${ }^{52}$. As GPR15 is involved in T cell homing and has been linked to colitis and inflammatory phenotypes, it is hypothesized to play a key role in smoking-related health risks ${ }^{53}$.

The PGS for another non-blood trait, educational attainment ${ }^{54}$, correlated significantly with the expression of 21 genes (FDR<0.05; Figure 6E, Supplementary Table 15). Several of the strongly associated genes are known to be involved in neuronal processes (Supplementary Table 19) and show expression in neuronal tissues (GTEx v7, Extended Data Figure 15). STX1B (strongest eQTS $P=1.3 \times 10^{-20}$ ) is specifically expressed in brain, and its encoded protein, syntaxin $1 \mathrm{~B}$, participates in the exocytosis of synaptic vesicles and synaptic transmission ${ }^{55}$. Another gene highly expressed in brain, $L R R N 3$ (Leucine-rich repeat neuronal protein 3; strongest eQTS $\mathrm{P}=1.7 \times 10^{-11}$ ) was negatively associated with the PGS for educational attainment, and has been associated with autism susceptibility ${ }^{56}$. The downregulated NRG1 (neuregulin 1; strongest eQTS $\mathrm{P}=4.5 \times 10^{-7}$ ), 
encodes a well-established growth factor involved in neuronal development and has been associated to synaptic plasticity ${ }^{57}$. NRG1 was also positively associated with the PGS for monocyte levels $^{20}$ (strongest eQTS $P=1.5 \times 10^{-7}$ ), several LDL cholesterol traits (e.g. medium LDL particles ${ }^{44}$; strongest eQTS $P=6.2 \times 10^{-8}$ ), coronary artery disease ${ }^{33}$ (strongest eQTS $P=1.5 \times 10^{-6}$ ) and body mass index in females ${ }^{58}$ (strongest eQTS $P=9.2 \times 10^{-12}$ ).

eQTS can also identify pathways known to be associated with monogenic diseases. For example, the PGSs for serine, glycine, the glycine derivative n-acetylglycine and creatine ${ }^{59,60}$ (Figure 6C) were all negatively associated with the gene expression levels of $P H G D H, P S A T 1$ and AARS (P < $\left.5.3 \times 10^{-7}\right) . P H G D H$ and PSAT1 encode crucial enzymes that regulate the synthesis of serine and, in turn, glycine ${ }^{61}$ (Figure 6D), while n-acetylglycine and creatine form downstream of glycine ${ }^{62}$. Mutations in PSAT1 and PHGDH can result in serine biosynthesis defects including phosphoserine aminotransferase deficiency ${ }^{63}$, phosphoglycerate dehydrogenase deficiency ${ }^{64}$, and Neu-Laxova syndrome ${ }^{65}$, all diseases characterized by low concentrations of serine and glycine in blood and severe neuronal manifestations. AARS encodes alanyl-tRNA synthetase, which links alanine to tRNA molecules. A mutation in AARS has been linked to Charcot Marie Tooth disease ${ }^{66}$, while the phenotypically similar hereditary sensory neuropathy type $1\left(\mathrm{HSN} 1^{67}\right)$ can be caused by a mutation in the gene encoding serine palmitoyltransferase. The gene facilitates serine's role in sphingolipid metabolism ${ }^{68}$. Disturbances in this pathway are hypothesized to be central in the development the neuronal symptoms ${ }^{69}$, suggesting a link between $A A R S$ expression and the serine pathway. Unexpectedly, the genetic risk for higher levels of these amino acids was associated with lower expression of $P H G D H, P S A T 1$, and $A A R S$, implying the presence of a negative feedback loop that controls serine synthesis. 
We next evaluated 6 immune diseases for which sharing of loci has been reported previously, and also observed sharing of downstream eQTS effects for these diseases (Supplementary Table 20). For example, the interferon gene STAT1 was significantly associated with T1D, celiac disease $(\mathrm{CeD}), \mathrm{IBD}$ and primary biliary cirrhosis (PBC). However, some of these genes are also marker genes for specific blood-cell types, such as $C D 79 A$, which showed a significant correlation with T1D and PBC. To test whether disease-specific eQTS gene signatures are reflected by blood cell proportions, we investigated single-cell RNA-seq data ${ }^{31}$ (Online Methods; Figure 6G). For ulcerative colitis (a subtype of IBD), we observed significant depletion of expression in megakaryocytes. SLE eQTS genes were enriched for antigen presentation (GeneNetwork $P=1.3 \times 10^{-5}$ ) and interferon signaling (GeneNetwork $P=1.4 \times 10^{-4}$ ), consistent with the well-described interferon signature in SLE patients ${ }^{70,71}$. Moreover, the SLE genes were significantly enriched for expression in mature dendritic cells, whose maturation depends on interferon signaling ${ }^{72}$. For CeD, we observed strong depletion of eQTS genes in monocytes and dendritic cells, and a slight enrichment in CD4+ and CD8+ T cells. The enrichment of cytokine (GeneNetwork $P=1.6 \times 10^{-15}$ ) and interferon (GeneNetwork $P=7.8 \times 10^{-13}$ ) signaling among the CeD eQTS genes is expected as a result of increased $\mathrm{T}$ cell populations.

\section{Cell-type-specificity of eQTS associations}

We next ascertained to what extent these eQTS associations can be replicated in non-blood tissues. We therefore aimed to replicate the significant eQTS effects in 1,460 LCL samples and 762 iPSC samples. Due to the fact these cohorts have a comparatively low sample sizes and study different cell types, we observed limited replication: 10 eQTS showed significant replication effect $($ FDR $<0.05)$ in the LCL dataset, with 9 out of those $(90 \%)$ showing the same effect direction as in 
the discovery set (Extended Data Figure 16A, Supplementary Table 17). For iPSCs, only 5 eQTS showed a significant effect (Extended Data Figure 16B, Supplementary Table 17). Since only a few eQTS associations are significant in non-blood tissues and the majority of identified eQTS associations are for blood-related traits, we speculate these effects are likely to be highly cell-type specific. This indicates that large-scale eQTL meta-analyses in other tissues could uncover more genes on which trait-associated SNPs converge.

\section{Discussion}

We here performed cis-eQTL, trans-eQTL and eQTS analyses in 31,684 blood samples, reflecting a six-fold increase over earlier large-scale studies ${ }^{1,5}$. We identified cis-eQTL effects for $88.3 \%$ and trans-eQTL effects for $32 \%$ of all genes that are expressed in blood.

We observed that cis-eQTL SNPs map close to the TSS or TES of the cis-gene: for the top $20 \%$ strongest cis-eQTL genes, $84.1 \%$ of the lead eQTL SNPs map within $20 \mathrm{~kb}$ of the gene, indicating that these are variants immediately adjacent to the start or end of transcripts that primarily drive cis-eQTL effects. The trait-associated variants that we studied showed a different pattern: $77.4 \%$ map within $20 \mathrm{~kb}$ of the closest protein-coding gene, suggesting that the genetic architecture of ciseQTLs is different from disease-associated variants. This is supported by the epigenetic differences that we observed between these two groups and can also partly explain, why we did not observe significantly increased overlap between genes prioritized using pathway enrichment analysis ${ }^{15}$ and genes prioritized using our cis-eQTLs. 
In contrast, for numerous traits we observed that multiple unlinked trans-eQTL variants often converge on genes with a known role in the biology for these traits (e.g. the involvement of interferon genes in SLE).

We therefore focused on trans-eQTL and eQTS results to gain insight into trait-relevant genes and pathways (Figures 4, 6). We estimate that $17.4 \%$ of our trans-eQTLs are driven by transcriptional regulation, whereas the remaining fraction is driven by not-yet-identified mechanisms. Our results support a model which postulates that, compared to cis-eQTLs, weaker distal and polygenic effects converge on core (key driver) genes that are more relevant to the traits and more specific for traitrelevant cell types (Figure 1B). The examples we have highlighted demonstrate how insights can be gained from our resource, and we envision similar interpretation strategies can be applied to the other identified trans-eQTL and eQTS effects. The catalog of genetic effects on gene expression we present here (available at www.eqtlgen.org) is a unique compendium for the development and application of novel methods that prioritize causal genes for complex traits $^{14,73}$, as well as for interpreting the results of genome-wide association studies. 


\section{Methods}

\section{Cohorts}

eQTLGen Consortium data consists of 31,684 blood and PBMC samples from 37 datasets, preprocessed in a standardized way and analyzed by each cohort analyst using the same settings (Online Methods). 26,886 (85\%) of the samples added to discovery analysis were whole blood samples and 4,798 (15\%) were PBMCs, and the majority of samples were of European ancestry (Supplementary Table 1). The gene expression levels of the samples were profiled by Illumina $(\mathrm{N}=17,421 ; 55 \%)$, Affymetrix U291 ( $\mathrm{N}=2,767 ; 8.7 \%)$, Affymetrix HuEx v1.0 ST (N=5,075; 16\%) expression arrays and by RNA-seq $(\mathrm{N}=6,422 ; 20.3 \%)$. A summary of each dataset is outlined in Supplementary Table 1. Detailed cohort descriptions can be found in the Supplementary Note. Each of the cohorts completed genotype and expression data pre-processing, PGS calculation, cis-eQTL-, trans-eQTL- and eQTS-mapping, following the steps outlined in the online analysis plans, specific for each platform (see URLs) or with slight alterations as described in Supplementary Table 1 and the Supplementary Note. All but one cohort (Framingham Heart Study), included non-related individuals into the analysis.

\section{Genotype data preprocessing}

The primary pre-processing and quality control of genotype data was conducted by each cohort, as specified in the original publications and in the Supplementary Note. The majority of cohorts used genotypes imputed to $1 \mathrm{kG}$ p1v3 or a newer reference panel. GenotypeHarmonizer ${ }^{74}$ was used to harmonize all genotype datasets to match the GIANT 1 kG p1v3 ALL reference panel and 
to fix potential strand issues for A/T and C/G SNPs. Each cohort tested SNPs with minor allele frequency $(\mathrm{MAF})>0.01$, Hardy-Weinberg P-value $>0.0001$, call rate $>0.95$, and $\mathrm{MACH} \mathrm{r}^{2}>0.5$.

\section{Expression data preprocessing}

\section{Illumina arrays}

Illumina array datasets expression were profiled by HT-12v3, HT-12v4 and HT-12v4 WGDASL arrays. Before analysis, all the probe sequences from the manifest files of those platforms were remapped to GRCh37.p10 human genome and transcriptome, using SHRiMP v2.2.3 aligner ${ }^{75}$ and allowing 2 mismatches. Probes mapping to multiple locations in the genome were removed from further analyses.

For Illumina arrays, the raw unprocessed expression matrix was exported from GenomeStudio. Before any pre-processing, the first two principal components (PCs) were calculated on the expression data and plotted to identify and exclude outlier samples. The data was normalized in several steps: quantile normalization, $\log _{2}$ normalization, probe centering and scaling by the

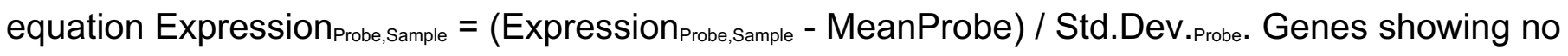
variance were removed. Next, the first four multidimensional scaling (MDS) components, calculated based on non-imputed and pruned genotypes using plink $v 1.07^{76}$, were regressed out of the expression matrix to account for population stratification. We further removed up to 20 first expression-based PCs that were not associated to any SNPs, as these capture non-genetic variation in expression. Each cohort also ran MixupMapper ${ }^{77}$ software to identify incorrectly labeled genotype-expression combinations, and to remove identified sample mix-ups. 


\section{Affymetrix arrays}

Affymetrix array-based datasets used the expression data previously pre-processed and quality controlled as indicated in the Supplementary Note.

\section{RNA-seq}

Alignment, initial quality control and quantification differed slightly across datasets, as described in the Supplementary Note. Each cohort removed outliers as described above, and then used Trimmed Mean of M-values (TMM) normalization and a counts per million (CPM) filter to include genes with $>0.5$ CPM in at least $1 \%$ of the samples. Other steps were identical to Illumina processing, with some exceptions for the BIOS Consortium datasets (Supplementary Note).

\section{Cis-eQTL mapping}

Cis-eQTL mapping was performed in each cohort using a pipeline described previously ${ }^{1}$. In brief, the pipeline takes a window of $1 \mathrm{Mb}$ upstream and $1 \mathrm{Mb}$ downstream around each SNP to select genes or expression probes to test, based on the center position of the gene or probe. The associations between these SNP-gene combinations was calculated using a Spearman correlation. Next, 10 permutation rounds were performed by shuffling the links between genotype and expression identifiers and re-calculating associations. The false discovery rate (FDR) was determined using 10 meta-analyzed permutations: for each gene in the real analysis, the most significant association was recorded, and the same was done for each of the permutations, 
resulting in a gene-level FDR. Cis-eQTLs with a gene-level FDR $<0.05$ (corresponding to $P<$ $\left.1.829 \times 10^{-5}\right)$ and tested in at least two cohorts were deemed significant.

\section{Trans-eQTL mapping}

Trans-eQTL mapping was performed using a previously described pipeline ${ }^{1}$ while testing a subset of 10,317 SNPs previously associated with complex traits. We required the distance between the SNP and the center of the gene or probe to be $>5 \mathrm{Mb}$. To maximize the power to identify transeQTL effects, the results of the summary statistics based or iterative conditional cis-eQTL mapping analyses (Supplementary Note) were used to correct the expression matrices before trans-eQTL mapping. For that, top SNPs for significant conditional cis-eQTLs were regressed out from the expression matrix. Finally, we removed potential false positive trans-eQTLs caused by reads crossmapping with cis regions (Supplementary Note).

\section{Genetic risk factor selection}

Genetic risk factors were downloaded from three public repositories: the EBI GWAS Catalogue ${ }^{78}$ (downloaded 21.11.2016), the NIH GWAS Catalogue and Immunobase (www.immunobase.org; accessed 26.04.2016), applying a significance threshold of $P \leq 5 \times 10^{-8}$. Additionally, we added 2,706 genome-wide significant GWAS SNPs from a recent blood trait GWAS ${ }^{20}$. SNP coordinates were lifted to hg19 using the liftOver command from R package rtracklayer $\mathrm{v} 1.34 .1^{79}$ and subsequently standardized to match the GIANT 1kG p1v3 ALL reference panel. This yielded 10,562 SNPs (Supplementary Table 2). We tested associations between all risk factors and 
genes that were at least $5 \mathrm{Mb}$ away to ensure that that they did not tag a cis-eQTL effect. All together, 10,317 trait-associated SNPs were tested in trans-eQTL analyses.

\section{eQTS mapping}

PGS trait inclusion

Full association summary statistics were downloaded from several publicly available resources

(Supplementary Table 13). GWAS performed exclusively in non-European cohorts were omitted.

Filters applied to the separate data sources are indicated in the Supplementary Note. All the dbSNP rs numbers were standardized to match GIANT $1 \mathrm{kG} p 1 \mathrm{v} 3$, and the directions of effects were standardized to correspond to the GIANT 1kG p1v3 minor allele. SNPs with opposite alleles compared to GIANT alleles were flipped. SNPs with A/T and C/G alleles, tri-allelic SNPs, indels, SNPs with different alleles in GIANT 1 kG p1v3 and SNPs with unknown alleles were removed from the analysis. Genomic control was applied to all the P-values for the datasets not genotyped by Immunochip or Metabochip. Additionally, genomic control was skipped for one dataset that did not have full associations available ${ }^{80}$ and for all the datasets from the GIANT consortium, as for these genomic control had already been applied. All together, 1,263 summary statistic files were added to the analysis. Information about the summary statistics files can be found in the Supplementary Note and Supplementary Table 14.

\section{PGS calculation}

A custom Java program, GeneticRiskScoreCalculator-v0.1.0c, was used for calculating several PGS in parallel. Independent effect SNPs for each summary statistics file were identified by double- 
clumping by first using a $250 \mathrm{~kb}$ window and subsequently a $10 \mathrm{Mb}$ window with LD threshold $R^{2}=0.1$. Subsequently, weighted PGS were calculated by summing the risk alleles for each independent SNP, weighted by its GWAS effect size (beta or log(OR) from the GWAS study). Four GWAS P-value thresholds $\left(P<5 \times 10^{-8}, 1 \times 10^{-5}, 1 \times 10^{-4}\right.$ and $\left.1 \times 10^{-3}\right)$ were used for constructing PGS for each summary statistics file.

\section{Pruning the SNPs and PGS}

To identify a set of independent genetic risk factors, we conducted LD-based pruning as implemented in PLINK $1.9^{81}$ with the setting --indep-pairwise 5050.1 . This yielded in 4,586 uncorrelated SNPs $\left(R^{2}<0.1\right.$, GIANT 1kG p1v3 ALL).

To identify the set of uncorrelated PGS, ten permuted trans-eQTL Z-score matrices from the combined trans-eQTL analysis were first confined to the pruned set of SNPs. Those matrices were then used to identify 3,042 uncorrelated genes, based on Z-score correlations (absolute Pearson $R<0.05)$. Next, permuted eQTS Z-score matrices were confined to uncorrelated genes and used to calculate pairwise correlations between all genetic risk scores to define a set of 1,873 uncorrelated genetic risk scores (Pearson $\left.\mathrm{R}^{2}<0.1\right)$.

\section{Empirical probe matching}

To integrate different expression platforms (four different Illumina array models, RNA-seq, Affymetrix U291 and Affymetrix Hu-Ex v1.0 ST) for the purpose of meta-analysis, we developed an empirical probe-matching approach. We used the pruned set of SNPs to conduct per-platform meta-analyses for all Illumina arrays, for all RNA-seq datasets, and for each Affymetrix dataset separately, using summary statistics from analyses without any gene expression correction for 
principal components. For each platform, this yielded an empirical trans-eQTL Z-score matrix, as well as ten permuted Z-score matrices, where links between genotype and expression files were permuted. Those permuted Z-score matrices reflect the gene-gene or probe-probe correlation structure.

We used RNA-seq permuted Z-score matrices as a gold standard reference and calculated for each gene the Pearson correlation coefficients with all the other genes, yielding a correlation profile for each gene. We then repeated the same analysis for the Illumina meta-analysis, and the two different Affymetrix platforms. Finally, we correlated the correlation profiles from each array platform with the correlation profiles from RNA-seq. For each array platform, we selected the probe showing the highest Pearson correlation with the corresponding gene in the RNA-seq data and treated those as matching expression features in the combined meta-analyses. This yielded 19,960 genes that were detected in RNA-seq datasets and tested in the combined meta-analyses. Genes and probes were matched to Ensembl v7182 (see URLs) stable gene IDs and HGNC symbols in all the analyses.

\section{Cross-platform replications}

To test the performance of the empirical probe-matching approach, we conducted discovery cis-, trans- and eQTS meta-analyses for each expression platform (RNA-seq, Illumina, Affymetrix U291 and Affymetrix Hu-Ex v1.0 ST arrays; array probes matched to 19,960 genes by empirical probe matching). For each discovery analysis, we conducted replication analyses in the three remaining platforms, observing strong replication of both cis-eQTLs, trans-eQTLs and eQTS in different platforms, with very good concordance in allelic direction. 


\section{Meta-analyses}

We meta-analyzed the results using a weighted Z-score method $^{1}$, where the Z-scores are weighted by the square root of the sample size of the cohort. For cis-eQTL and trans-eQTL meta-analyses, this resulted in a final sample size of $\mathrm{N}=31,684$. The combined eQTS meta-analysis included the subset of unrelated individuals from the Framingham Heart Study, resulting in a combined sample size of 28,158 .

\section{Quality control of the meta-analyses}

For quality control of the overall meta-analysis results, MAFs for all tested SNPs were compared between eQTLGen and 1kG p1v3 EUR (Extended Data Figure 3), and the effect direction of each dataset was compared against the meta-analyzed effect (Extended Data Figure 2A-C).

\section{FDR calculation for trans-eQTL and eQTS mapping}

To determine nominal P-value thresholds corresponding to FDR $=0.05$, we used the pruned set of SNPs for trans-eQTL mapping and permutation-based FDR calculation, as described previously ${ }^{1}$. We leveraged those results to determine the P-value threshold corresponding to FDR $=0.05$ and used this as a significance level in trans-eQTL mapping in which all 10,317 genetic trait-associated SNPs were tested. In the eQTS analysis, an analogous FDR calculation was performed using a pruned set of PGSs. We analyzed only SNP/PGS-gene pairs tested in at least two cohorts. 


\section{Positive and negative set of trans-eQTLs}

Based on the results of integrative trans-eQTL mapping, we defined true positive (TP) and true negative $(\mathrm{TN})$ sets of trans-eQTLs. TP set was considered as all significant $(\mathrm{FDR}<0.05)$ transeQTLs. TN set of trans-eQTLs was selected as non-significant (max absolute meta-analysis Zscore 3; all FDR>0.05) SNP-gene combinations, adhering to following conditions:

1. The size of TN set was set equal to the size of TP set (59,786 trans-eQTLs).

2. Each SNP giving trans-eQTL effects on $X$ genes in the TP set, is also giving trans-eQTL effects on $\mathrm{X}$ genes in the TN set.

3. Each gene that is affected in trans by Y SNPs in the TP set, is also affected in trans by $Y$ SNPs in the TN set.

4. Adhere to the correlation structure of the SNPs: if two SNPs are in perfect LD, they affect the same set of genes, both in the TP set and in the TN set.

5. Adhere to the correlation structure of the genes: if two genes are perfectly co-expressed, they are affected by the same SNPs, both in the TP set and in the TN set.

This set of TN trans-eQTLs was used in subsequent enrichment analyses as the matching set for comparison.

\section{Conditional trans-eQTL analyses}

We aimed to estimate how many trans-eQTL SNPs were likely to drive both the trans-eQTL effect and the GWAS phenotype. The workflow of this analysis is shown in Extended Data Figure 6. We 
used the integrative trans-eQTL analysis results as an input, confined ourselves to those effects which were present in the datasets we had direct access to (BBMRI-BIOS+EGCUT; $N=4,339)$, and showed nominal $\mathrm{P}<8.3115 \times 10^{-06}$ in the meta-analysis of those datasets. This $\mathrm{P}$-value threshold was the same as in the full combined trans-eQTL meta-analysis and was based on the FDR=0.05 significance threshold identified from the analysis run on the pruned set of GWAS SNPs after removal of cross-mapping effects. We used the same methods and SNP filters as in the full combined trans-eQTL meta-analysis, aside from the FDR calculation, which was based on the full set of SNPs, instead of the pruned set of SNPs.

For each significant trans-eQTL SNP, we defined the locus by adding a $\pm 1 \mathrm{Mb}$ window around it. Next, for each trans-eQTL gene we ran iterative conditional trans-eQTL analysis using all loci for given trans-eQTL gene. We then evaluated the LD between all conditional top trans-eQTL SNPS and GWAS SNPs using a $1 \mathrm{Mb}$ window and $\mathrm{R}^{2}>0.8$ (1kG p1v3 EUR) as a threshold for LD overlap.

\section{Trans-eQTL mediation analysis}

To identify potential mediators of trans-eQTL effects we used a $G \times E$ interaction model: $\mathrm{t}=\beta_{0}+\beta_{1} \times \mathrm{s}+\beta_{2} \times \mathrm{m}+\beta_{3} \times \mathrm{s} \times \mathrm{m}$

Where $\mathrm{t}$ is the expression of the trans-eQTL gene, $\mathrm{s}$ is the trans-eQTL SNP, and $\mathrm{m}$ is the expression of a potential mediator gene within $100 \mathrm{~kb}$ of the trans-eQTL SNP. On top of the gene expression normalization that we used for the rest of our analysis, we used a rank-based inverse normal transformation to enforce a normal distribution before fitting the linear model, identical to the normalization used by Zhernakova et al. ${ }^{47}$ in their $\mathrm{G} \times \mathrm{E}$ interaction $\mathrm{eQTL}$ analyses. We fitted this model separately on each of the cohorts that are part of the BIOS consortium. We transformed the interaction P-values to Z-scores and used the weighted Z-score method ${ }^{83}$ to perform a meta- 
analysis on the in total 3,831 samples. The Benjamini \& Hochberg procedure ${ }^{84}$ was used to limit the FDR to 0.05. The plots in Extended Data Figure 9 are created with the default normalization, the regression lines are the best-fitting lines between the mediator gene and the trans eQTL gene,

stratified by genotype. We used a Fisher's exact test to calculate the enrichment of significant (FDR $\leq 0.05)$ interactions between our TP trans-eQTLs and the interactions identified in the TN transeQTL set.

\section{TF and tissue enrichment analyses}

We downloaded the curated sets of known TF targets and tissue-expressed genes from the Enrichr web site ${ }^{85,86}$. TF target gene sets included TF targets as assayed by ChIP-X experiments from ChEA $^{87}$ and ENCODE ${ }^{88,89}$ projects, and tissue-expressed genes were based on the ARCHS4 database ${ }^{90}$. Those gene sets were used to conduct hypergeometric over-representation analyses as implemented into the $\mathrm{R}$ package ClusterProfiler ${ }^{91}$.

\section{SMR analyses}

To gain further insight into genes that are important in the biology of the trait, we used the combined cis-eQTL results to perform $\mathrm{SMR}^{14}$ for 16 large GWAS studies (Supplementary Table 20). We derived cis-eQTL beta and standard error of the beta (SE(beta)) from the Z-score and the MAF reported in $1 \mathrm{kG}$ v1p3 ALL, using the following formulae ${ }^{14}$

beta $=z /\left(\sqrt{ }\left(2 p(1-p)\left(n+z^{2}\right)\right)\right.$ 
$\operatorname{SE}($ beta $)=1 /\left(\sqrt{ }\left(2 p(1-p)\left(n+z^{2}\right)\right)\right.$

Where $\mathrm{p}$ is the MAF and $\mathrm{n}$ is the sample size.

The cis-eQTLs were converted to the dense BESD format. The $1 \mathrm{kG} p 1 \mathrm{v} 3 \mathrm{ALL}$ reference panel was also used to calculate LD, and SMR analysis was run using the SMR software v0.706 without any P-value cut-offs on either GWAS or eQTL input.

\section{DEPICT}

We applied DEPICT v194 ${ }^{15}$ to the same 16 recent GWAS traits as above (Supplementary Table 20), using all variants that attain a genome-wide significant P-value threshold. Specifically, we looked at the gene prioritization and gene set enrichment analyses to compare the results with the output of other prioritization methods (SMR $\left.{ }^{14}\right)$.

\section{Comparison of gene prioritization with DEPICT and SMR}

To investigate the consistency between results from two gene prioritization methods, we compared the enrichment of overlapping genes for 16 GWAS traits (Supplementary Table 20). We confined ourselves to genes that were tested in SMR and that fell within the DEPICT loci, and tested whether genes significant in SMR (P-value $<0.05 /$ number of tested genes) and DEPICT $($ FDR $<0.05)$ were enriched (one-sided Fisher's Exact Test). 


\section{Epigenetic marks enrichment}

We ascertained epigenetic properties of the lead cis-eQTL SNPs, and contrasted these to a set of 3,688 trait-associated SNPs that were associated with either blood-related traits (such as mean corpuscular volume or platelet counts) or immune-mediated diseases. The SNPs were annotated with histone and chromatin marks information from the Epigenomics Roadmap Project. We summarized the information by calculating the overlap ratio across 127 human cell types between the epigenetic marks and the SNP within a window size of $+/-25 \mathrm{bp}$ : if a SNP co-localizes with a mark for all 127 cell-types, the score for that SNP will be 1; if a SNP co-localizes with a mark for none of the cell-types, the score will be 0 .

The reason we chose only SNPs associated to blood-related traits and immune-mediated diseases was to minimize potential confounding due to a subtle bias in the Epigenomics Roadmap Project towards blood cell-types: 29 of the 127 cell-types that we studied were blood cell types. However, when redoing the epigenetic enrichment analysis, while excluding these blood cell types, we did not see substantial differences in the enriched and depleted histone marks.

\section{Chromosomal contact analyses}

\section{Capture Hi-C overlap for cis-eQTLs}

To assess whether cis-eQTL lead SNPs overlapped with chromosomal contact as measured using $\mathrm{Hi}-\mathrm{C}$ data, we used promoter capture $\mathrm{Hi}-\mathrm{C}$ data ${ }^{92}$, downloaded from $\mathrm{CHiCP}^{93}$ (see URLs). We took the lead eQTL SNPs and overlapped these with the capture Hi-C data and studied the 10,428 ciseQTL genes for which this data is available. We then checked whether the Capture Hi-C target 
maps within $5 \mathrm{~kb}$ of the lead SNP. Of 508 cis-eQTL genes that mapped over $100 \mathrm{~kb}$ from the TSS or TES, 223 overlapped capture Hi-C data (27.8\%). Of 7,984 cis-eQTL genes that mapped within $100 \mathrm{~kb}$ from the TSS or TES, 1,641 overlapped capture Hi-C data $\left(17.0 \%, \mathrm{Chi}^{2}\right.$ test $\left.\mathrm{P}=10^{-14}\right)$. To ensure this was not an artefact, we performed the same analysis, while flipping the location of the capture $\mathrm{Hi}-\mathrm{C}$ target with respect to the location of the bait, and did not observe any significant difference $\left(\mathrm{Chi}^{2}\right.$ test $\left.\mathrm{P}=0.59\right)$.

\section{Hi-C overlap enrichment analysis for trans-eQTLs}

To assess whether trans-eQTLs were enriched for chromosomal contacts as measured using HiC data, we downloaded the contact matrices for the human lymphoblastoid GM12878 cell line ${ }^{19}$ (GEO accession GSE63525). We used the intrachromosomal data at a resolution of 10kb with mapping quality of 30 or more (MAPQGE30), and normalized using the KRnorm vectors. For each of the 59,786 trans-eQTLs, we evaluated whether any contact was reported in this dataset. We divided each trans-eQTL SNP and any of their proxies $\left(R^{2}>0.8,1 \mathrm{kG} p 1 \mathrm{v} 3, \mathrm{EUR}\right.$, acquired from SNiPA 94 ; URLs) in 10kb blocks. The trans-eQTL genes were also assigned to $10 \mathrm{~kb}$ blocks, and to multiple blocks if the gene was more than $10 \mathrm{~kb}$ in length (length between TSS and TES, Ensembl v71). For each individual trans-eQTL SNP-gene pair, we then determined if there was any overlap with the Hi-C contact matrices. We repeated this analysis using the true negative set of transeQTLs described before to generate a background distribution of expected contact.

\section{Data availability}

Full summary statistics from eQTLGen meta-analyses are available on the eQTLGen website: www.eqtlgen.org which was built using the MOLGENIS framework ${ }^{95}$. 


\section{Code availability}

Individual cohorts participating in the study followed the analysis plans as specified in the URLs or with slight alterations as described in the Methods and Supplementary Note. All tools and source code, used for genotype harmonization, identification of sample mixups, eQTL mapping, metaanalyses and for calculating polygenic scores are freely available at https://github.com/molgenis/systemsgenetics/.

\section{Acknowledgments}

The cohorts participating in this study list the acknowledgments in the cohort-specific supplemental information in Supplementary Note.

We thank i2QTL CONSORTIUM for providing the iPSC replication results.

We thank Kate McIntyre for editing the final text.

This work is supported by a grant from the European Research Council (ERC Starting Grant agreement number 637640 ImmRisk) to LF and a VIDI grant (917.14.374) from the Netherlands Organisation for Scientific Research (NWO) to LF. We thank the UMCG Genomics Coordination Center, MOLGENIS team, the UG Center for Information Technology, and the UMCG research IT program and their sponsors in particular BBMRI-NL for data storage, high performance compute and web hosting infrastructure. BBMRI-NL is a research infrastructure financed by the Netherlands Organization for Scientific Research (NWO) [grant number 184.033.111]. 


\section{URLS}

Full summary statistics from this study, www.eqtlgen.org

ExAC pLI scores, http://exac.broadinstitute.org/downloads;

Ensembl v71 annotation file,

ftp://ftp.ensembl.org/pub/release-71/gtf/homo sapiens;

Reference for genotype harmonizing,

ftp://share.sph.umich.edu/1000genomes/fullProject/2012.03.14/GIANT.phase1_release_v3.2010

1123.snps_indels_svs.genotypes.refpanel.ALL.vcf.gz.tgz

eQTLGen analysis plan for Illumina array datasets,

https://github.com/molgenis/systemsgenetics/wiki/eQTL-mapping-analysis-cookbook;

eQTLGen analysis plan for RNA-seq datasets,

https://github.com/molgenis/systemsgenetics/wiki/eQTL-mapping-analysis-cookbook-for-RNA-

seq-data;

eQTLGen analysis plan for Affymetrix array datasets,

https://github.com/molgenis/systemsgenetics/wiki/QTL-mapping-analysis-cookbook-for-

Affymetrix-expression-arrays;

GenotypeHarmonizer, https://github.com/molgenis/systemsgenetics/wiki/Genotype-Harmonizer;

Protocol to resolve sample mixups, https://github.com/molgenis/systemsgenetics/wiki/Resolving-

$\underline{\text { mixups; }}$

Enrichr gene set enrichment libraries,

http://amp.pharm.mssm.edu/Enrichr/;

GeneOverlap package for enrichment analyses,

https://www.bioconductor.org/packages/release/bioc/html/GeneOverlap.html; 
SHRiMP aligner used for re-mapping Illumina probes,

http://compbio.cs.toronto.edu/shrimp/;

EBI GWAS Catalogue,

https://www.ebi.ac.uk/gwas/;

Immunobase,

http://www.immunobase.org/;

ClusterProfiler package used for tissue enrichment analyses,

http://bioconductor.org/packages/release/bioc/html/clusterProfiler.html;

Capture Hi-C data,

https://www.chicp.org/

SNiPA, used to acquire proxy SNPs,

http://snipa.helmholtz-muenchen.de/snipa3/

Regulatory Circuits, used to acquire TF data,

www.RegulatoryCircuits.org 


\section{References:}

1. Westra, H.-J. et al. Systematic identification of trans eQTLs as putative drivers of known disease associations. Nat. Genet. 45, 1238-1243 (2013).

2. Kirsten, $\mathrm{H}$. et al. Dissecting the genetics of the human transcriptome identifies novel traitrelated trans-eQTLs and corroborates the regulatory relevance of non-protein coding loci†. Hum. Mol. Genet. 24, 4746-4763 (2015).

3. Lloyd-Jones, L. R. et al. The Genetic Architecture of Gene Expression in Peripheral Blood. Am. J. Hum. Genet. 100, 228-237 (2017).

4. Jansen, R. et al. Conditional eQTL analysis reveals allelic heterogeneity of gene expression. Hum. Mol. Genet. 26, 1444-1451 (2017).

5. Joehanes, R. et al. Integrated genome-wide analysis of expression quantitative trait loci aids interpretation of genomic association studies. Genome Biol. 18, 16 (2017).

6. Brynedal, B. et al. Large-Scale trans-eQTLs Affect Hundreds of Transcripts and Mediate Patterns of Transcriptional Co-regulation. Am. J. Hum. Genet. 100, 581-591 (2017).

7. Yao, C. et al. Dynamic Role of trans Regulation of Gene Expression in Relation to Complex Traits. Am. J. Hum. Genet. 100, 571-580 (2017).

8. Boyle, E. A., Li, Y. I. \& Pritchard, J. K. An Expanded View of Complex Traits: From Polygenic to Omnigenic. Cell 169, 1177-1186 (2017).

9. Lewis, C. M. \& Vassos, E. Prospects for using risk scores in polygenic medicine. Genome Med. 9, 96 (2017).

10. Natarajan, P. et al. Polygenic Risk Score Identifies Subgroup With Higher Burden of Atherosclerosis and Greater Relative Benefit From Statin Therapy in the Primary Prevention 
Setting. Circulation 135, 2091-2101 (2017).

11. Lek, M. et al. Analysis of protein-coding genetic variation in 60,706 humans. Nature $\mathbf{5 3 6}$, 285-291 (2016).

12. Wu, Y., Zheng, Z., Visscher, P. M. \& Yang, J. Quantifying the mapping precision of genomewide association studies using whole-genome sequencing data. Genome Biol. 18, 86 (2017).

13. Melé, M. et al. The human transcriptome across tissues and individuals. Science $\mathbf{3 4 8 , 6 6 0 -}$ 665 (2015).

14. Zhu, Z. et al. Integration of summary data from GWAS and eQTL studies predicts complex trait gene targets. Nat. Genet. 48, 481-487 (2016).

15. Pers, T. H. et al. Biological interpretation of genome-wide association studies using predicted gene functions. Nat. Commun. 6, 5890 (2015).

16. Giambartolomei, C. et al. Bayesian test for colocalisation between pairs of genetic association studies using summary statistics. PLoS Genet. 10, e1004383 (2014).

17. Marbach, D. et al. Tissue-specific regulatory circuits reveal variable modular perturbations across complex diseases. Nat. methods 13, 366-370 (2016).

18. Li, T. et al. A scored human protein-protein interaction network to catalyze genomic interpretation. Nat. methods 14, 61-64 (2017).

19. Rao, S. S. P. et al. A 3D map of the human genome at kilobase resolution reveals principles of chromatin looping. Cell 159, 1665-1680 (2014).

20. Astle, W. J. et al. The Allelic Landscape of Human Blood Cell Trait Variation and Links to Common Complex Disease. Cell 167, 1415-1429.e19 (2016).

21. Perry, J. R. et al. Parent-of-origin-specific allelic associations among 106 genomic loci for age at menarche. Nature 514, 92-97 (2014). 
22. Ding, Y. et al. ZNF131 suppresses centrosome fragmentation in glioblastoma stem-like cells through regulation of HAUS5. Oncotarget 8, 48545-48562 (2017).

23. Oh, Y. \& Chung, K. C. Small ubiquitin-like modifier (SUMO) modification of zinc finger protein 131 potentiates its negative effect on estrogen signaling. J. Biol. Chem. 287, 17517-17529 (2012).

24. Lemaitre, R. N. et al. Genetic loci associated with plasma phospholipid n-3 fatty acids: a meta-analysis of genome-wide association studies from the CHARGE Consortium. PLoS Genet. 7, e1002193 (2011).

25. Deelen, P. et al. Improving the diagnostic yield of exome-sequencing, by predicting genephenotype associations using large-scale gene expression analysis. bioRxiv preprint (2018).

26. Plagnol, V. et al. Genome-wide association analysis of autoantibody positivity in type 1 diabetes cases. PLoS Genet. 7, e1002216 (2011).

27. Liu, J. Z. et al. Association analyses identify 38 susceptibility loci for inflammatory bowel disease and highlight shared genetic risk across populations. Nat. Genet. 47, 979-986 (2015).

28. Gateva, V. et al. A large-scale replication study identifies TNIP1, PRDM1, JAZF1, UHRF1BP1 and IL10 as risk loci for systemic lupus erythematosus. Nat. Genet. 41, 12281233 (2009).

29. Yin, X. et al. Genome-wide meta-analysis identifies multiple novel associations and ethnic heterogeneity of psoriasis susceptibility. Nat. Commun. 6, 6916 (2015).

30. Moffatt, M. F. et al. A large-scale, consortium-based genomewide association study of asthma. New Engl. J. Med. 363, 1211-1221 (2010).

31. van der Wijst, M. G. P. et al. Single-cell RNA sequencing identifies celltype-specific cis- 
eQTLs and co-expression QTLs. Nat. Genet. 50, 493-497 (2018).

32. Wang, J. H. et al. Aiolos regulates B cell activation and maturation to effector state. Immunity 9, 543-553 (1998).

33. Nikpay, M. et al. A comprehensive 1,000 Genomes-based genome-wide association metaanalysis of coronary artery disease. Nat. Genet. 47, 1121-1130 (2015).

34. Wood, A. R. et al. Defining the role of common variation in the genomic and biological architecture of adult human height. Nat. Genet. 46, 1173-1186 (2014).

35. He, M. et al. Meta-analysis of genome-wide association studies of adult height in East Asians identifies 17 novel loci. Hum. Mol. Genet. 24, 1791-1800 (2015).

36. Schoenherr, C. J. \& Anderson, D. J. The neuron-restrictive silencer factor (NRSF): a coordinate repressor of multiple neuron-specific genes. Science 267, 1360 LP-1363 (1995).

37. Chong, J. A. et al. REST: a mammalian silencer protein that restricts sodium channel gene expression to neurons. Cell 80, 949-957 (1995).

38. Cheong, A. et al. Downregulated REST transcription factor is a switch enabling critical potassium channel expression and cell proliferation. Mol. cell 20, 45-52 (2005).

39. Dibner, C., Schibler, U. \& Albrecht, U. The mammalian circadian timing system: organization and coordination of central and peripheral clocks. Annu. Rev. Physiol. 72, 517-549 (2010).

40. Bass, J. \& Lazar, M. A. Circadian time signatures of fitness and disease. Sci. 354, 994-999 (2016).

41. Song, C. et al. REV-ERB agonism suppresses osteoclastogenesis and prevents ovariectomy-induced bone loss partially via FABP4 upregulation. FASEB J. : Off. Publ. Fed. Am. Soc. Exp. Biol. 32, 3215-3228 (2018).

42. Liu, X., Li, Y. I. \& Pritchard, J. K. Trans effects on gene expression can drive omnigenic 
inheritance. bioRxiv (2018).

43. Teslovich, T. M. et al. Biological, clinical and population relevance of 95 loci for blood lipids. Nature 466, 707-713 (2010).

44. Willer, C. J. et al. Discovery and refinement of loci associated with lipid levels. Nat. Genet. 45, 1274-1283 (2013).

45. Wang, X. et al. Macrophage ABCA1 and ABCG1, but not SR-BI, promote macrophage reverse cholesterol transport in vivo. J. Clin. Investig. 117, 2216-2224 (2007).

46. Goldstein, J. L. \& Brown, M. S. Binding and degradation of low density lipoproteins by cultured human fibroblasts. Comparison of cells from a normal subject and from a patient with homozygous familial hypercholesterolemia. J. Biol. Chem. 249, 5153-5162 (1974).

47. Zhernakova, D. V. et al. Identification of context-dependent expression quantitative trait loci in whole blood. Nat. Genet. 49, 139-145 (2017).

48. Singh, A. B., Kan, C. F. K., Shende, V., Dong, B. \& Liu, J. A novel posttranscriptional mechanism for dietary cholesterol-mediated suppression of liver LDL receptor expression. J. Lipid Res. 55, 1397-1407 (2014).

49. Tobacco and Genetics Consortium. Genome-wide meta-analyses identify multiple loci associated with smoking behavior. Nat. Genet. 42, 441-447 (2010).

50. Kõks, S. \& Kõks, G. Activation of GPR15 and its involvement in the biological effects of smoking. Exp. Biol. Med. 242, 1207-1212 (2017).

51. van Iterson, M., van Zwet, E. W., BIOS Consortium \& Heijmans, B. T. Controlling bias and inflation in epigenome- and transcriptome-wide association studies using the empirical null distribution. Genome Biol. 18, 19 (2017).

52. Bauer, M., Fink, B., Seyfarth, H.-J., Wirtz, H. \& Frille, A. Tobacco-smoking induced GPR15- 
expressing T cells in blood do not indicate pulmonary damage. BMC Pulm. Med. 17, 159 (2017).

53. Kõks, G. et al. Smoking-Induced Expression of the GPR15 Gene Indicates Its Potential Role in Chronic Inflammatory Pathologies. Am. J. Pathol. 185, 2898-2906 (2015).

54. Okbay, A. et al. Genome-wide association study identifies 74 loci associated with educational attainment. Nature 533, 539-542 (2016).

55. Smirnova, T., Miniou, P., Viegas-Pequignot, E. \& Mallet, J. Assignment of the human syntaxin 1B gene (STX) to chromosome $16 \mathrm{p} 11.2$ by fluorescence in situ hybridization. Genomics 36, 551-553 (1996).

56. Sousa, I. et al. Polymorphisms in leucine-rich repeat genes are associated with autism spectrum disorder susceptibility in populations of European ancestry. Mol. Autism 1, 7 (2010).

57. Agarwal, A. et al. Dysregulated expression of neuregulin-1 by cortical pyramidal neurons disrupts synaptic plasticity. Cell reports 8, 1130-1145 (2014).

58. Locke, A. E. et al. Genetic studies of body mass index yield new insights for obesity biology. Nature 518, 197-206 (2015).

59. Kettunen, J. et al. Genome-wide study for circulating metabolites identifies 62 loci and reveals novel systemic effects of LPA. Nat. Commun. 7, 11122 (2016).

60. Shin, S.-Y. et al. An atlas of genetic influences on human blood metabolites. Nat. Genet. 46, 543-550 (2014).

61. El-Hattab, A. W. Serine biosynthesis and transport defects. Mol. Genet. Metab. 118, 153159 (2016).

62. Leuzzi, V., Alessandrì, M. G., Casarano, M., Battini, R. \& Cioni, G. Arginine and glycine 
stimulate creatine synthesis in creatine transporter 1-deficient lymphoblasts. Anal. Biochem. 375, 153-155 (2008).

63. Hart, C. E. et al. Phosphoserine aminotransferase deficiency: a novel disorder of the serine biosynthesis pathway. Am. J. Hum. Genet. 80, 931-937 (2007).

64. Klomp, L. W. et al. Molecular characterization of 3-phosphoglycerate dehydrogenase deficiency--a neurometabolic disorder associated with reduced L-serine biosynthesis. Am. J. Hum. Genet. 67, 1389-1399 (2000).

65. Shaheen, R. et al. Neu-Laxova syndrome, an inborn error of serine metabolism, is caused by mutations in PHGDH. Am. J. Hum. Genet. 94, 898-904 (2014).

66. McLaughlin, H. M. et al. A recurrent loss-of-function alanyl-tRNA synthetase (AARS) mutation in patients with Charcot-Marie-Tooth disease type 2N (CMT2N). Hum. Mutat. 33, 244-253 (2012).

67. Auer-Grumbach, M. Hereditary sensory neuropathy type I. Orphanet J. rare Dis. 3, 7 (2008).

68. Hanada, K. Serine palmitoyltransferase, a key enzyme of sphingolipid metabolism. Biochim. et Biophys. Acta 1632, 16-30 (2003).

69. Glinton, K. E. et al. Disturbed phospholipid metabolism in serine biosynthesis defects revealed by metabolomic profiling. Mol. Genet. Metab. 123, 309-316 (2018).

70. Baechler, E. C. et al. Interferon-inducible gene expression signature in peripheral blood cells of patients with severe lupus. Proc. Natl. Acad. Sci. United States Am. 100, 2610-2615 (2003).

71. Bennett, L. et al. Interferon and granulopoiesis signatures in systemic lupus erythematosus blood. J. Exp. Med. 197, 711-723 (2003).

72. Pantel, A. et al. Direct type I IFN but not MDA5/TLR3 activation of dendritic cells is required 
for maturation and metabolic shift to glycolysis after poly IC stimulation. PLoS Biol. 12, e1001759 (2014)

73. Hormozdiari, F. et al. Colocalization of GWAS and eQTL Signals Detects Target Genes. Am. J. Hum. Genet. 99, 1245-1260 (2016).

74. Deelen, P. et al. Genotype harmonizer: automatic strand alignment and format conversion for genotype data integration. BMC Res. notes 7, 901 (2014).

75. Rumble, S. M. et al. SHRiMP: accurate mapping of short color-space reads. PLoS Comput. Biol. 5, e1000386 (2009).

76. Purcell, S. et al. PLINK: a tool set for whole-genome association and population-based linkage analyses. Am. J. Hum. Genet. 81, 559-575 (2007).

77. Westra, H.-J. et al. MixupMapper: correcting sample mix-ups in genome-wide datasets increases power to detect small genetic effects. Bioinforma. 27, 2104-2111 (2011).

78. MacArthur, J. et al. The new NHGRI-EBI Catalog of published genome-wide association studies (GWAS Catalog). Nucleic acids Res. 45, D896-D901 (2017).

79. Lawrence, M., Gentleman, R. \& Carey, V. rtracklayer: an R package for interfacing with genome browsers. Bioinformatics 25, (2009).

80. Hyde, C. L. et al. Identification of 15 genetic loci associated with risk of major depression in individuals of European descent. Nat. Genet. 48, 1031-1036 (2016).

81. Chang, C. C. et al. Second-generation PLINK: rising to the challenge of larger and richer datasets. GigaScience 4, 7 (2015).

82. Zerbino, D. R. et al. Ensembl 2018. Nucleic acids Res. 46, D754-D761 (2018).

83. Zaykin, D. V. Optimally weighted Z-test is a powerful method for combining probabilities in meta-analysis. J. Evol. Biol. 24, 1836-1841 (2011). 
84. Benjamini, Y. \& Hochberg, Y. Controlling the False Discovery Rate: A Practical and Powerful Approach to Multiple Testing: a practical and powerful approach to multiple testing. J. $R$. Stat. Soc. Ser. B 57, 289-300 (1995).

85. Chen, E. Y. et al. Enrichr: interactive and collaborative HTML5 gene list enrichment analysis tool. BMC Bioinforma. 14, 128 (2013).

86. Kuleshov, M. V. et al. Enrichr: a comprehensive gene set enrichment analysis web server 2016 update. Nucleic acids Res. 44, W90-W97 (2016).

87. Lachmann, A. et al. ChEA: transcription factor regulation inferred from integrating genomewide ChIP-X experiments. Bioinforma. 26, 2438-2444 (2010).

88. ENCODE Project Consortium. The ENCODE (ENCyclopedia Of DNA Elements) Project. Sci. 306, 636-640 (2004).

89. ENCODE Project Consortium. A user's guide to the encyclopedia of DNA elements (ENCODE). PLoS Biol. 9, e1001046 (2011).

90. Lachmann, A. et al. Massive mining of publicly available RNA-seq data from human and mouse. Nat. Commun. 9, 1366 (2018).

91. Yu, G., Wang, L.-G., Han, Y. \& He, Q.-Y. clusterProfiler: an R package for comparing biological themes among gene clusters. Omics : J. Integr. Biol. 16, 284-287 (2012).

92. Javierre, B. M. et al. Lineage-Specific Genome Architecture Links Enhancers and Noncoding Disease Variants to Target Gene Promoters. Cell 167, 1369-1384.e19 (2016).

93. Schofield, E. C. et al. CHiCP: a web-based tool for the integrative and interactive visualization of promoter capture Hi-C datasets. Bioinforma. 32, 2511-2513 (2016).

94. Arnold, M., Raffler, J., Pfeufer, A., Suhre, K. \& Kastenmuller, G. SNiPA: an interactive, genetic variant-centered annotation browser. Bioinformatics 31, (2015). 
bioRxiv preprint doi: https://doi.org/10.1101/447367; this version posted October 19, 2018. The copyright holder for this preprint (which was not certified by peer review) is the author/funder. All rights reserved. No reuse allowed without permission.

95. Swertz, M. et al. The MOLGENIS toolkit: rapid prototyping of biosoftware at the push of a button. BMC Bioinforma. 11, 12 (2010). 